\title{
Generation of highly diverse peptide library by linear-double-stranded DNA based AND gate
}

\section{genetic circuit in mammalian cells}

\author{
Shuai $\mathrm{Li}^{1,2 \# *}$, Weijun $\mathrm{Su}^{3 \#}$, Chunze Zhang ${ }^{4}$ \\ 1. Department of Breast Cancer Pathology and Research Laboratory, Tianjin Medical University \\ Cancer Institute and Hospital, National Clinical Research Center for Cancer; Key Laboratory of \\ Cancer Prevention and Therapy, Tianjin; Tianjin's Clinical Research Center for Cancer, 300060,
}

China;

2. State Key Laboratory of Medicinal Chemical Biology, Nankai University, Tianjin 300071, China;

3. School of Medicine, Nankai University, Tianjin 300071, China;

4. Department of Colorectal Surgery, Tianjin Union Medical Center, Tianjin 300121, China.

\# Shuai Li and Weijun Su contributed equally to this work.

* To whom correspondence should be addressed. Tel: +86 22 23340123; Fax: +86 22 23340123;

Email: shuaili@tmu.edu.cn \& shuai.li2001@gmail.com. 


\section{Abstract}

DNA-encoded peptide libraries are ideal functional peptide discovery platforms for their extremely

large capacity. However, it's still difficult to build high content peptide library in intact mammalian

cells, which offer advantages associated with appropriate protein modification, proper protein folding, and natural status of membrane protein. Our previous work established linear-double-stranded DNAs (ldsDNAs) as innovative biological parts to implement AND gate genetic circuits in mammalian cell line. In the current study, we employ ldsDNA with terminal NNK degenerate codons as AND gate

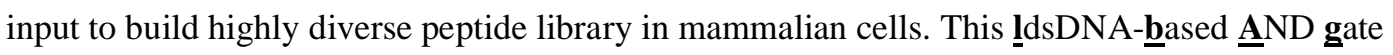
(LBAG) peptide strategy is easy to conduct, only PCR reaction and cell transfection experiments are needed. High-throughput sequencing (HTS) results reveal that our new LBAG strategy could generate peptide library with both amino acid sequence and peptide length diversities. Moreover, by a mammalian cell two-hybrid system, we pan an MDM2 protein interacting peptide through the LBAG peptide library. Our work establishes ldsDNA as biological parts for building highly diverse peptide library in mammalian cells.

\section{Keywords}

peptide library, linear-double-stranded DNA (ldsDNA), AND gate, degenerate codon. 


\section{Introduction}

Different state-of-the-art display technologies, e.g., phage, ribosome, mRNA, bacterial, and yeast-display, have been developed to identify functional peptides (1-7). Through construction and screen vast peptide libraries, these display technologies physically couple between phenotype (high affinity high selectivity binding) and genotype (DNA sequence). Currently, plasmids and PCR products have been explored as genetic mediums to store highly diverse coding information of the peptide library $(8,9)$. These genetic materials are introduced into phage-host bacteria, yeast, or test tubes to transcript-translate into peptides. However, it's still a big challenge to build high content peptide library in intact mammalian cells. Some attempts have been made to introduce DNA-encoded peptide library into mammalian cells using episomal-, viral- or transposon-mediated gene transfer (10-17). While, the peptide library capacity is constantly lost during plasmid extraction, lentivirus generation, transfection/infection process. This leads to small library size relative to other display technologies.

We previously applied linear-double-stranded DNA (ldsDNA, or PCR amplicon) as novel biological parts to implement Boolean logic AND gate genetic circuits in mammalian cells (18). Via splitting essential gene expression cassette into signal-mute ldsDNA, our strategy achieves two or three-input AND gate calculation with a low noise-signal ratio both in vitro and in vivo. This split-relink process is similar to $\mathrm{V}(\mathrm{D}) \mathrm{J}$ recombination, which serves as the fundamental molecular mechanism to generate highly diverse repertoires of antigen-receptors (antibodies and T-cell receptors)

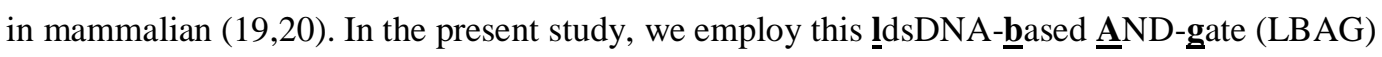
genetic circuit to generate high content peptide library. 


\section{Results}

\section{Generation of peptide library by ldsDNA-based AND-gate genetic circuit}

Our previous study demonstrated that ldsDNAs (PCR amplicons), split from intact gene expression cassettes (containing promoter, gene coding sequence and polyA signal), could undergo reconnection to form one output-signal-generating molecule in cultured cells (18). Here we hypothesize that ldsDNAs with terminal NNK degenerate codons could generate highly diverse peptide library through IdsDNA- $\underline{\text { based }}$ AND-gate (LBAG) calculation in mammalian cells (Figure 1).

Taking pEGFP-C1 plasmid as PCR template, primers with multiple tandem NNK-trinucleotides were used to generate ldsDNAs with terminal degenerate codons (Supplementary Table 1). Three kinds of ldsDNAs, containing CMV promoter, Kozak sequence and different number of NNK-trinucleotides, were produced (namely CMV-Kozak- $\langle\mathrm{NNK}\rangle_{5}, \mathrm{CMV}-$ Kozak- $<\mathrm{NNK}>_{10}$, CMV-Kozak- $\langle\mathrm{NNK}\rangle_{15}$ ) (for sequences see Supplementary Materials). Meanwhile, two kinds of ldsDNAs, containing terminal NNK-trinucleotides (or not), GFP coding sequence and SV40 polyA signal (PAS), were amplified (namely $\langle\mathrm{NNK}\rangle_{5}$-GFP-SV40 PAS, $\langle\mathrm{NNK}\rangle_{0}$-GFP-SV40 PAS). Six different combinations of these two subgroup ldsDNAs were introduced into HEK293T cells to construct the AND-gate genetic circuits. The six combinations are as following: 1, CMV-Kozak- $\langle\text { NNK }\rangle_{5}$ with $\langle\mathrm{NNK}\rangle_{5}$-GFP-SV40 PAS $(5 \mathrm{X}+5 \mathrm{X}$ for short $) ; 2$, CMV-Kozak- $\langle\mathrm{NNK}\rangle_{10}$ with $\langle\text { NNK }\rangle_{5}$-GFP-SV40 PAS $(10 \mathrm{X}+5 \mathrm{X}) ; 3, \mathrm{CMV}-$ Kozak- $\langle\mathrm{NNK}\rangle_{15}$ with $\langle\mathrm{NNK}\rangle_{5}$-GFP-SV40 PAS $(15 \mathrm{X}+5 \mathrm{X}) ; 4, \mathrm{CMV}-$ Kozak- $<\mathrm{NNK}\rangle_{5}$ with $\langle\mathrm{NNK}\rangle_{0}$-GFP-SV40 PAS $(5 \mathrm{X}+0 \mathrm{X}) ; 5$, 
CMV-Kozak- $\langle\text { NNK }\rangle_{10}$ with $\langle\text { NNK }\rangle_{0}$-GFP-SV40 PAS $(10 \mathrm{X}+0 \mathrm{X}) ; 6, \mathrm{CMV}-$ Kozak- $\langle\text { NNK }\rangle_{15}$ with $<$ NNK $\rangle_{0}$-GFP-SV40 PAS $(15 \mathrm{X}+0 \mathrm{X})$.

When AND-gate genetic circuits are built up in cells, peptide library would be generated surrounding the junctions formed between these two subtype ldsDNAs (Figure 1). Forty-eight hours after transfection, total RNAs were extracted then cDNAs were synthesized via reverse transcription. Nucleotide sequences surrounding the junctions formed by two ldsDNA-inputs were PCR amplified and then subjected to pair-end high-throughput sequencing (HTS). The HTS statistics for all sequencing libraries are shown in Table 1.

\section{Reading frame phase of IdsDNA-based peptide library}

To display or tether library peptides, oligopeptides are usually fused to specific anchor proteins. For example in phage display experiment, the peptide library is fused to an open reading frame (ORF) thereby connecting peptide to phage coat protein (1). Our previous research demonstrated that the ldsDNA could be subject to terminal nucleotide(s) deletion which may lead to reading frame shift. Here we analyze the reading frame phase characteristics of the LBAG peptide libraries (Figure 2). Libraries generated by dual-side $\left({ }_{2} \mathrm{X}+\mathrm{X}\right.$ ) terminal-NNKs-ldsDNAs, e.g., CMV-Kozak- $\langle\mathrm{NNK}\rangle_{5}$ with $\langle\mathrm{NNK}\rangle_{5}$-GFP-SV40 PAS $(5 \mathrm{X}+5 \mathrm{X})$, show a similar reading frame phase distribution. The average in-frame $(3 \mathrm{n})$ ratio ranging from $45 \%$ to $47 \%$. While libraries generated by single-side ( _ X+0X ) terminal-NNKs-ldsDNAs, e.g., CMV-Kozak- $\langle\mathrm{NNK}\rangle_{5}$ with $\langle\mathrm{NNK}\rangle_{0}-\mathrm{GFP}-\mathrm{SV} 40$ PAS $(5 \mathrm{X}+0 \mathrm{X})$, show dramatic dynamics of reading frame phase distribution (Figure 2$) .5 \mathrm{X}+0 \mathrm{X}$ peptide libraries reach the maximum $69 \%$ in-frame rate compared with $63 \%$ of $10 \mathrm{X}+0 \mathrm{X}$ and $53 \%$ of $15 \mathrm{X}+0 \mathrm{X}$, 
respectively. Then, the in-frame nucleotide sequences (3n) are translated into polypeptides for further analysis.

\section{Length dynamic and amino acid composition of IdsDNA-based peptide library}

We next analyze the polypeptide length distributions of different LBAG peptide libraries (peptides with pre-stop codon are excluded). Dual-side terminal-NNKs-ldsDNAs strategies, including 5X+5X, $10 \mathrm{X}+5 \mathrm{X}$ and $15 \mathrm{X}+5 \mathrm{X}$, show a more dynamic length distribution compared with that from single-side terminal-NNKs-ldsDNAs strategies (5X+0X, 10X+0X and 15X+0X) (Figure 3). In addition, for single-side terminal-NNKs-ldsDNAs strategies, most peptides have a length equal to the theoretical polypeptide length generated by direct ligation (supposing no insertion or deletion happened) (Figure $3 \mathrm{D}-\mathrm{F})$. For example, in the $5 \mathrm{X}+0 \mathrm{X}$ group, over $81 \%$ of the peptide length is five amino acids.

The amino acid composition of different LBAG peptide libraries is in line with the theoretical NNK amino acid composition (pre-stop codon is included in the analysis) (Table 2). However, Proline (P) residue always shows a higher percentage than the theoretical ratio, particularly when the peptide chain getting longer. Meanwhile, the percentage of each nucleotide at each position of peptide library codons is showed in Supplementary Table 2. The nucleotide distributions are in line with NNK degenerate base ratio.

\section{The capacity of the LBAG peptide library}

Peptide library capacity could be represented by its coverage ability on amino acid combination possibilities. Here, all eighteen LBAG peptide libraries cover all 400 possible dual-amino-acid combinations (2-mer). For triple-amino-acid (3-mer), the library coverage is ranging from $64 \%$ to $98 \%$ (Figure 4A). For quadruple-amino-acid (4-mer), the library coverage is ranging from 5.7\% to $32.1 \%$ 
(Figure 4B). The peptide library content, defined as the total amino acid number coded by a library, serves as a determinate factor of library capacity (Figure 4).

\section{The asymmetry of input IdsDNA end processing during AND gate calculation}

As shown by Figure 2 and 3, single-side ( _X+OX ) and dual-side ( _X+_X ) LBAG peptide library strategies show obvious differences in both reading frame phase distributions and peptide length dynamics. To further investigate these differences and figure out whether or not the up-stream and down-stream ldsDNAs have the same chance of end processing, we PCR-generated two pairs of AND gate ldsDNAs: pair 1, CMV-Kozak-FLAG-NNN with GFP-SV40 PAS; and pair 2,

CMV-Kozak-FLAG with NNN-GFP-SV40 PAS (N for randomized nucleotides, for ldsDNA sequences see Supplementary Materials). After cell transfection, RNA extraction, cDNA synthesis, and PCR amplification, high-throughput sequencing (HTS) was performed to characterize the oligonucleotide sequences formed by AND gate calculation. About $67 \%$ of the sequences formed by CMV-Kozak-FLAG-NNN and GFP-SV40 PAS contains intact random nucleotides (NNN) (Figure 5). While for CMV-Kozak-FLAG and NNN-GFP-SV40 PAS group, about 57\% of oligonucleotide contains triple nucleotides (Figure 5). Thus, the down-stream ldsDNAs (NNN-GFP-SV40 PAS) are more likely subject to end processing than up-stream ldsDNAs (CMV-Kozak-FLAG-NNN) in AND gate relink process in cells. This may be the reason why single-side ( $\left.\_\mathrm{X}+0 \mathrm{X}\right)$ strategy generate higher in-frame rate and less variability among the peptide length than dual-side ( _ X+_X ) strategy.

In addition, through the above terminal-triple-random-nucleotide-ldsDNA AND gate calculation, we also evaluated the nucleotide preferences of LBAG calculation. In both CMV-Kozak-FLAG-NNN + GFP-SV40 PAS group and CMV-Kozak-FLAG + NNN-GFP-SV40 PAS group, Guanine (G) is 
always the unfavorable end nucleotide (Supplementary Table 3). Furthermore, up-stream or down-stream ldsDNAs containing two or three terminal Guanines are hard to connect to the corresponding ldsDNAs (Supplementary Table 3).

\section{Using LBAG peptide library to identify MDM2 protein interacting peptide}

The E3 ubiquitin ligase MDM2 negatively regulates the activity of the tumor suppressor protein p53 through interacting with the N-terminal transactivation domain of p53. Structurally, the residues F19, W23 and L26 in p53, which insert deep into the MDM2 hydrophobic cleft, are critical in MDM2-p53 interaction (21). Also, the non-peptide inhibitors of the p53-MDM2 interaction have to contain mimics of these amino acids (22-24). Pazgier et al. screened a duodecimal peptide phage display library to identify MDM2 (residues 25-109) interacting peptides (25). They found 14 out of 15 MDM2 binding

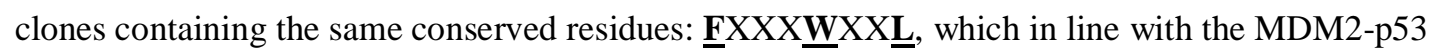
binding pattern. Shiheido et al. employed mRNA display to identify MDM2 interacting peptides (26,27). Similarly, they found more than half of all peptides retained the three hydrophobic residues corresponding to F19, W23 and L26 of wild-type p53. Here, to test the performance of our LBAG peptide library strategy, we plan to identify the MDM2 interacting peptides in mammalian cells.

To detect protein-protein interaction in mammalian cells, we employed the two-hybrid system, which has been adapted for use in mammalian cells $(28,29)$. The system contains three plasmids: pACT, which expresses VP16 activation domain, pBIND, which expresses GAL4 DNA-binding domain, pG5egfp, which contains five GAL4 binding sites upstream of a minimal TATA box and EGFP ORF. In the present study, MDM2 (residues 25-109) is subcloned into pACT plasmid (namely pACT-MDM2). By PCR amplification, the LBAG peptide library is fused to GAL4 DNA-binding 
domain through an XTEN linker (Figure 6A). To achieve high library content, we here used single-side LBAG strategy.

Two plasmids (pACT-MDM2, pG5egfp) and two ldsDNAs (CMV-GAL4-XTEN- $\left\langle\right.$ NNK ${ }_{15}$, SV40 PAS) were cotransfected into HEK293T cells. The peptide library will be formed through the AND gate calculation between CMV-GAL4-XTEN- $\langle\text { NNK }\rangle_{15}$ and SV40 PAS (Figure 6A). If the peptide interacts with MDM2, the activation domain VP16 will be recruited to GAL4 binding sites, thereby activating the GFP reporter gene expression (Figure 6B). Here fluorescence-activated cell sorting (FACS) was used to isolate the GFP positive cells. High-throughput sequencing of the peptide library in these cells identified peptide SEVIRWTALWPGPRS among the top hits (23 reads, rank fourth)

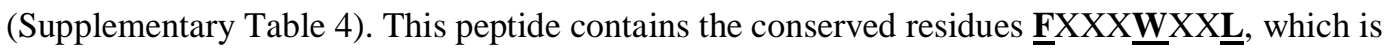
critical to interact with MDM2.

\section{Discussion}

Affinity-based display technologies, e.g., phage, ribosome, mRNA, bacterial, and yeast-display, have been developed to screen peptide libraries to identify protein-binding peptides. Peptide library size is a determinate factor of a success screen. For phage and yeast-display, the complexity of peptide library stores in plasmid $(1,2,7)$. Currently, the phage display library size could reach $10^{9}(9,30)$. While for ribosome and mRNA-display, PCR products serve as genetic medium to store library coding information. Ribosome and mRNA-display could achieve extremely large library size $\left(>10^{13}\right)(5,31)$. In the current research, we provide a new strategy to build high content peptide library in cultured cell line. The protocol is easy to conduct, only containing two steps: PCR amplification and cell 
transfection. Since degenerate trinucleotides is present at the 5' end of the primer, the library complexity keeps on increasing during PCR amplification process. Theoretically, no specific nucleotide sequence is enriched during PCR reaction. PCR products are then transfected into cells to conduct AND-gate linkage to generate full gene expression cassette (promoter-CDS-PAS). For our strategy, no particular attention needs to be paid to maintain the library abundance. The size of ldsDNA-based library needs to be further investigated. Determining the peptide sequence diversity in each cell by single-cell-sequencing will provide crucial information for estimating the whole library size.

For the current protocol, there are still several issues that need to be further addressed to increase library capacity. First of all, the high in-frame ratio would improve library capacity. Our results reveal that peptide libraries generated by single-side NNKs-ldsDNA show higher in-frame ratios than that from dual-side NNKs-ldsDNAs (Figure 2). Besides, chemical modified primers could be used to generate nuclease resistance ldsDNAs to prevent reading frame shift $(32,33)$. Another major obstacle is the pre-stop codon, which causes premature termination (34). For NNK degenerate codon, the percentage of stop-codon in each amino acid position is $3.125 \%(1 / 32)$ and will be accumulated when the peptide is getting longer. When the polypeptide length reaches 20 amino acids, about $47 \%$ of the sequences contain pre-stop codon. One potential solution to avoid stop codon is to synthesize PCR primer using trimer phosphoramidites $(35,36)$.

In the present study, we tested the feasibility of our LBAG peptide library strategy through the identification of the MDM2 interacting peptide (Figure 6). Indeed, one peptide

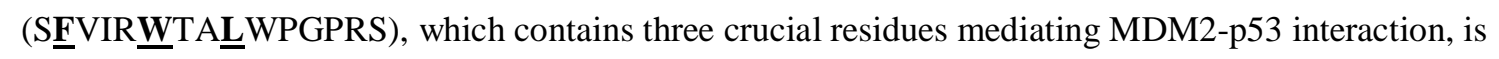
among the top hits (Supplementary Table 4). However, in phage display and mRNA display 


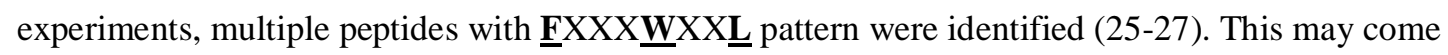
from the enrichment of interacting peptides during rounds of panning in phage and mRNA display process. For the current protocol, four components, including two plasmids (pACT-MDM2, pG5egfp) and two ldsDNAs (CMV-GAL4-XTEN- $\langle\mathrm{NNK}\rangle_{15}$, SV40 PAS), were transient cotransfected into one HEK293T cell to conduct AND gate calculation and assemble the two-hybrid system. In the future application, to save the cell transfection capacity, the plasmids should be preset into cells by generating a stable cell line. Thereby, only ldsDNAs need to be transfected into cells.

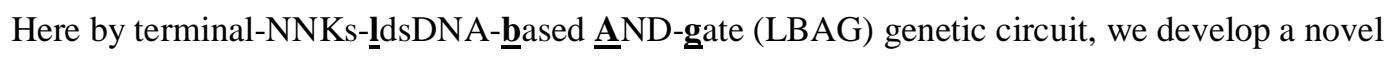
method to generate highly diverse peptide library in mammalian cells. Our protocol is easy to conduct only PCR-reaction and cell-transfection are needed. High-throughput sequencing results reveal that LBAG peptide library is characterized by both amino acids sequence diversity and peptide length dynamics. We also identified a peptide with conserved residues which are critical to bind to MDM2. Our new method may have great application potential in therapeutic peptide development.

\section{Materials and Methods}

\section{Cell culture}

The HEK293T cell line was maintained in Dulbecco's modified Eagle's medium (DMEM) (Thermo Fisher Scientific, Hudson, NH, USA) containing 10\% fetal bovine serum (FBS) with 1\% penicillin-streptomycin solution at 37 degree with $5 \% \mathrm{CO}_{2}$.

\section{IdsDNA synthesis}


KOD-Plus-Ver.2 DNA polymerase (Toyobo, Osaka, Japan) was used to amplify ldsDNAs (PCR amplicons) using the pEGFP-C1 (Clontech, Mountain View, CA, USA) plasmid as the template. PCR products underwent agarose electrophoresis and gel-purification to remove plasmid template and free dNTPs (Universal DNA purification kit, Tiangen, Beijing, China). The amount of PCR products was determined by the OD 260 absorption value. PCR primer sequences and ldsDNA sequences were present in Supplementary Table 1 and Supplementary Materials, respectively.

\section{IdsDNA transfection}

HEK293T cells were seeded into 6-well plates the day before transfection (60\% - 70\% confluency).

$500 \mathrm{ng} /$ well of each input amplicons (total $1000 \mathrm{ng} /$ well) were transfected into cells with

Lipofectamine 2000 (Invitrogen, Carlsbad, CA, USA) reagent following standard protocol.

\section{RNA extraction, cDNA synthesis and PCR amplification}

Forty-eight hours after transfection, total RNAs were extracted by RNAiso Plus (TaKaRa, Beijing,

China) reagent. cDNAs were synthesized using the PrimeScript ${ }^{\mathrm{TM}}$ RT reagent Kit with gDNA Eraser

(TaKaRa) following standard protocol. KOD-Plus-Ver.2 DNA polymerase (Toyobo) was used to amplify the corresponding cDNA sequences surrounding ldsDNA junctions.

\section{Two-hybrid system in mammalian cells}

The two-hybrid system used in this study was developed from commercial CheckMate ${ }^{\mathrm{TM}}$ Mammalian Two-Hybrid System (Promega, Madison, WI, USA) with some modifications. Briefly, MDM2 (residues 25-109) was subcloned into pACT (pACT empty vector, GenBank Accession Number AF264723) vector through XbaI and KpnI restriction sites (namely pACT-MDM2). EGFP sequence was cloned into pG5luc vector (pG5luc empty vector, GenBank Accession Number AF264724) to 
replace luciferase sequence through HindIII and FseI restriction sites (namely pG5egfp). Two IdsDNAs CMV-GAL4-XTEN- $\langle\mathrm{NNK}\rangle_{15}$ and SV40 PAS were PCR-generated using the pBIND (pBIND empty vector, GenBank Accession Number AF264722) plasmid as the template. pACT-MDM2 (500ng/well), pG5egfp (500ng/well), ldsDNA CMV-GAL4-XTEN- $\langle\text { NNK }\rangle_{15}$ (500ng/well) and ldsDNA SV40 PAS (250ng/well) were transient cotransfected into 6-well plated HEK293T cells using Lipofectamine 2000. The experiment could be scaled up by transfected more 6-well plated cells. Forty-eight hours after transfection, FACS was used to isolate the GFP positive cells.

\section{Library construction and high-throughput sequencing}

Library construction and high-throughput sequencing were conduct by Novogene (Beijing, China).

Sequencing libraries were generated using TruSeq ${ }^{\circledR}$ DNA PCR-Free Sample Preparation Kit (Illumina, San Diego, CA, USA) following the manufacturer's recommendations and index codes were added. The library quality was assessed on the Qubit Fluorometer and Agilent Bioanalyzer 2100 system. At last, the libraries were sequenced on an Illumina HiSeq 2500 platform and 250 bp paired-end reads were generated.

\section{Data analysis}

Paired-end reads from the original DNA fragments were merged using FLASH, a very fast and accurate analysis tool which was designed to merge paired-end reads when there are overlaps between reads 1 and reads 2. Paired-end reads were assigned to each sample according to the unique barcodes. Reads were filtered by QIIME quality filters. Nucleotide sequences generated by ldsDNA-based AND-gate genetic circuits were extracted by in-house Perl scripts. High-throughput sequencing data 
are deposited on the Gene Expression Omnibus (GEO, http://www.ncbi.nlm.nih.gov/geo) under the accession number GEO: GSE134671 (reviewer access token: kpujmequhnspngx).

\section{Acknowledgements}

This work was supported by the National Natural Science Foundation of China [31870860, 31400673

to S.L., 31971388, 81402407 to W.S.]; the Tianjin Research Program of Application Foundation and

Advanced Technology [14JCQNJC09800 to S.L., 15JCQNJC11700 to W.S.]; the State Key

Laboratory of Medicinal Chemical Biology (Nankai University) [2018103 to S.L.]; and the Key

Project of National Health and Family Planning Commission of Tianjin [2017057 to C.Z.]. The

authors have declared that no competing interest exists.

\section{Author Contributions}

S.L. conceived and supervised the study. S.L. and W.S. designed and performed the experiments. S.L. and W.S. analyzed the data. W.S. and S.L. prepared the figures. S.L. and W.S. wrote the paper. All authors discussed the results and reviewed the manuscript.

\section{Competing Interests}

The authors have declared that no competing interest exists. 


\section{Figure Legends}

Figure 1. Schematic showing the rationale of generating highly diverse peptide library by ldsDNA-based AND-gate (LBAG) calculation. ldsDNAs containing CMV promoter and tandem NNK degenerate codons are cotransfected with ldsDNAs containing NNK codons, GFP coding region, and polyA signal sequence. The AND-gate calculation is conducted between two kinds of ldsDNA to form peptide library.

Figure 2. Reading frame character of nucleotide sequences formed by different libraries. Pair-end high-throughput sequencing data is processed to extract the nucleotide sequences formed by ldsDNA-based AND-gate. The nucleotide sequence length is divided by three to show its reading frame character. The average percentage of different phase of different libraries is presented. All data are displayed as the mean $\pm \mathrm{SD}, \mathrm{n}=3$.

Figure 3. The peptide length distributions of six different peptide libraries generated by ldsDNA-based AND-gate genetic circuits. In-frame (3n) nucleotide sequences are translated into polypeptide sequences. Then the length of polypeptide sequence in each library is summarized. The average percentage of different polypeptide length is presented. All data are displayed as the mean \pm $\mathrm{SD}, \mathrm{n}=3$.

Figure 4. Peptide library coding capacity on covering triple-amino-acid (A) or quadruple-amino-acid (B) peptide possibilities. Peptide libraries are scanned by all triple or quadruple amino-acid combinations, respectively. The library content (defined as the total amino acid number coded by a library) and the percentage of coverage are presented.

Figure 5. Reading frame character of nucleotide sequences formed by ldsDNAs with terminal-triple-random-nucleotides. Two pairs of AND gate ldsDNAs were PCR-generated and transfected into HEK293T cells (pair 1, CMV-Kozak-FLAG-NNN with GFP-SV40 PAS; pair 2, CMV-Kozak-FLAG with NNN-GFP-SV40 PAS). Pair-end high-throughput sequencing data is processed to extract the nucleotide sequences formed by ldsDNA-based AND-gate. The average percentage of different phase of different libraries is presented. All data are displayed as the mean \pm $\mathrm{SD}, \mathrm{n}=4$.

Figure 6. Schematic showing the rationale of generating LBAG peptide library for two-hybrid system in mammalian cells. (A) ldsDNA CMV-GAL4-XTEN- $\langle\text { NNK }\rangle_{15}$ (containing CMV promoter, GAL4XTEN coding sequence, tandem NNK degenerate codons) is cotransfected with ldsDNA SV40 PAS. The AND-gate calculation is conducted between two ldsDNAs to form peptide library. (B) Schematic representation of the mammalian two-hybrid system. The pG5egfp plasmid contains five GAL4 binding sites upstream of a minimal TATA box, which in turn is upstream of the GFP gene. 
Interaction between the two test proteins, GAL4-peptide and MDM2-VP16, results in an expression of GFP protein.

\section{References}

1. Smith, G.P. (1985) Filamentous fusion phage: novel expression vectors that display cloned antigens on the virion surface. Science, 228, 1315-1317.

2. Scott, J.K. and Smith, G.P. (1990) Searching for peptide ligands with an epitope library. Science, 249, 386-390.

3. Mattheakis, L.C., Bhatt, R.R. and Dower, W.J. (1994) An in vitro polysome display system for identifying ligands from very large peptide libraries. Proceedings of the National Academy of Sciences of the United States of America, 91, 9022-9026.

4. Hanes, J. and Pluckthun, A. (1997) In vitro selection and evolution of functional proteins by using ribosome display. Proceedings of the National Academy of Sciences of the United States of America, 94, 4937-4942.

5. Roberts, R.W. and Szostak, J.W. (1997) RNA-peptide fusions for the in vitro selection of peptides and proteins. Proceedings of the National Academy of Sciences of the United States of America, 94, 12297-12302.

6. Georgiou, G., Stathopoulos, C., Daugherty, P.S., Nayak, A.R., Iverson, B.L. and Curtiss, R., 3rd. (1997) Display of heterologous proteins on the surface of microorganisms: from the screening of combinatorial libraries to live recombinant vaccines. Nat Biotechnol, 15, 29-34.

7. Boder, E.T. and Wittrup, K.D. (1997) Yeast surface display for screening combinatorial polypeptide libraries. Nat Biotechnol, 15, 553-557.

8. Henninot, A., Collins, J.C. and Nuss, J.M. (2018) The Current State of Peptide Drug Discovery:

Back to the Future? J Med Chem, 61, 1382-1414.

9. Obexer, R., Walport, L.J. and Suga, H. (2017) Exploring sequence space: harnessing chemical and biological diversity towards new peptide leads. Curr Opin Chem Biol, 38, 52-61.

10. Beerli, R.R., Bauer, M., Buser, R.B., Gwerder, M., Muntwiler, S., Maurer, P., Saudan, P. and Bachmann, M.F. (2008) Isolation of human monoclonal antibodies by mammalian cell display.

Proceedings of the National Academy of Sciences of the United States of America, 105, 14336-14341.

11. Waldmeier, L., Hellmann, I., Gutknecht, C.K., Wolter, F.I., Cook, S.C., Reddy, S.T., Grawunder, U. and Beerli, R.R. (2016) Transpo-mAb display: Transposition-mediated B cell display and functional screening of full-length IgG antibody libraries. mAbs, 8, 726-740.

12. Bowers, P.M., Horlick, R.A., Neben, T.Y., Toobian, R.M., Tomlinson, G.L., Dalton, J.L., Jones, H.A., Chen, A., Altobell, L., 3rd, Zhang, X. et al. (2011) Coupling mammalian cell surface display with somatic hypermutation for the discovery and maturation of human antibodies. Proceedings of the National Academy of Sciences of the United States of America, 108, 20455-20460.

13. Ho, M., Nagata, S. and Pastan, I. (2006) Isolation of anti-CD22 Fv with high affinity by Fv display on human cells. Proceedings of the National Academy of Sciences of the United States of America, 103, 9637-9642.

14. Zhang, H., Sturchler, E., Zhu, J., Nieto, A., Cistrone, P.A., Xie, J., He, L., Yea, K., Jones, T., Turn, R. et al. (2015) Autocrine selection of a GLP-1R G-protein biased agonist with potent antidiabetic effects. Nat Commun, 6, 8918. 
15. Xie, J., Zhang, H., Yea, K. and Lerner, R.A. (2013) Autocrine signaling based selection of combinatorial antibodies that transdifferentiate human stem cells. Proceedings of the National Academy of Sciences of the United States of America, 110, 8099-8104.

16. Stepanov, A.V., Markov, O.V., Chernikov, I.V., Gladkikh, D.V., Zhang, H., Jones, T., Sen'kova, A.V., Chernolovskaya, E.L., Zenkova, M.A., Kalinin, R.S. et al. (2018) Autocrine-based selection of ligands for personalized CAR-T therapy of lymphoma. Sci Adv, 4, eaau4580.

17. Zhang, H., Yea, K., Xie, J., Ruiz, D., Wilson, I.A. and Lerner, R.A. (2013) Selecting agonists from single cells infected with combinatorial antibody libraries. Chem Biol, 20, 734-741.

18. Li, S., Su, W. and Zhang, C. (2019) Linear double-stranded DNAs as innovative biological parts to implement genetic circuits in mammalian cells. FEBS J, 286, 2341-2354.

19. Bassing, C.H., Swat, W. and Alt, F.W. (2002) The mechanism and regulation of chromosomal V(D)J recombination. Cell, 109 Suppl, S45-55.

20. Schatz, D.G. (2004) V(D)J recombination. Immunol Rev, 200, 5-11.

21. Kussie, P.H., Gorina, S., Marechal, V., Elenbaas, B., Moreau, J., Levine, A.J. and Pavletich, N.P. (1996) Structure of the MDM2 oncoprotein bound to the p53 tumor suppressor transactivation domain. Science, 274, 948-953.

22. Chene, P. (2003) Inhibiting the p53-MDM2 interaction: an important target for cancer therapy. Nature reviews. Cancer, 3, 102-109.

23. Vassilev, L.T., Vu, B.T., Graves, B., Carvajal, D., Podlaski, F., Filipovic, Z., Kong, N., Kammlott, U., Lukacs, C., Klein, C. et al. (2004) In vivo activation of the p53 pathway by small-molecule antagonists of MDM2. Science, 303, 844-848.

24. Vassilev, L.T. (2007) MDM2 inhibitors for cancer therapy. Trends in molecular medicine, 13, 23-31.

25. Pazgier, M., Liu, M., Zou, G., Yuan, W., Li, C., Li, J., Monbo, J., Zella, D., Tarasov, S.G. and Lu, W. (2009) Structural basis for high-affinity peptide inhibition of p53 interactions with MDM2 and MDMX. Proceedings of the National Academy of Sciences of the United States of America, 106, 4665-4670.

26. Shiheido, H., Takashima, H., Doi, N. and Yanagawa, H. (2011) mRNA display selection of an optimized MDM2-binding peptide that potently inhibits MDM2-p53 interaction. PloS one, 6, e17898.

27. Nagata, T., Shirakawa, K., Kobayashi, N., Shiheido, H., Tabata, N., Sakuma-Yonemura, Y., Horisawa, K., Katahira, M., Doi, N. and Yanagawa, H. (2014) Structural basis for inhibition of the MDM2:p53 interaction by an optimized MDM2-binding peptide selected with mRNA display. PloS one, 9, e109163.

28. Dang, C.V., Barrett, J., Villa-Garcia, M., Resar, L.M., Kato, G.J. and Fearon, E.R. (1991)

Intracellular leucine zipper interactions suggest c-Myc hetero-oligomerization. Molecular and cellular biology, 11, 954-962.

29. Fearon, E.R., Finkel, T., Gillison, M.L., Kennedy, S.P., Casella, J.F., Tomaselli, G.F., Morrow, J.S. and Van Dang, C. (1992) Karyoplasmic interaction selection strategy: a general strategy to detect protein-protein interactions in mammalian cells. Proceedings of the National Academy of Sciences of the United States of America, 89, 7958-7962.

30. Smith, G.P. and Petrenko, V.A. (1997) Phage Display. Chem Rev, 97, 391-410.

31. Lamla, T. and Erdmann, V.A. (2003) Searching sequence space for high-affinity binding peptides using ribosome display. J Mol Biol, 329, 381-388. 
32. Rahal, E.A., Henricksen, L.A., Li, Y., Turchi, J.J., Pawelczak, K.S. and Dixon, K. (2008) ATM mediates repression of DNA end-degradation in an ATP-dependent manner. DNA Repair (Amst), 7 , 464-475.

33. Chang, H.H., Watanabe, G., Gerodimos, C.A., Ochi, T., Blundell, T.L., Jackson, S.P. and Lieber, M.R. (2016) Different DNA End Configurations Dictate Which NHEJ Components Are Most Important for Joining Efficiency. J Biol Chem, 291, 24377-24389.

34. Cho, G., Keefe, A.D., Liu, R., Wilson, D.S. and Szostak, J.W. (2000) Constructing high complexity synthetic libraries of long ORFs using in vitro selection. J Mol Biol, 297, 309-319.

35. Janczyk, M., Appel, B., Springstubbe, D., Fritz, H.J. and Muller, S. (2012) A new and convenient approach for the preparation of beta-cyanoethyl protected trinucleotide phosphoramidites. Org Biomol Chem, 10, 1510-1513.

36. Suchsland, R., Appel, B. and Muller, S. (2018) Preparation of trinucleotide phosphoramidites as synthons for the synthesis of gene libraries. Beilstein J Org Chem, 14, 397-406. 

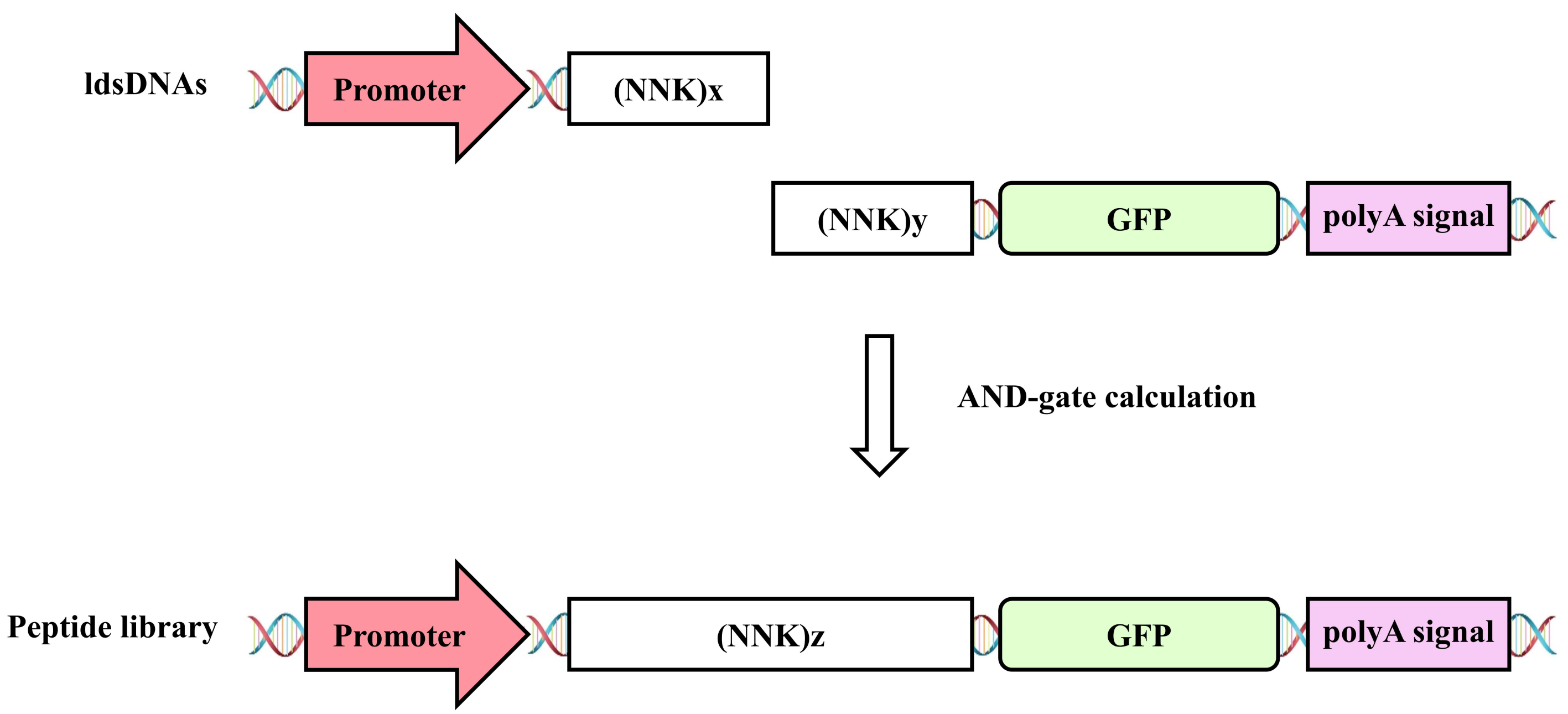


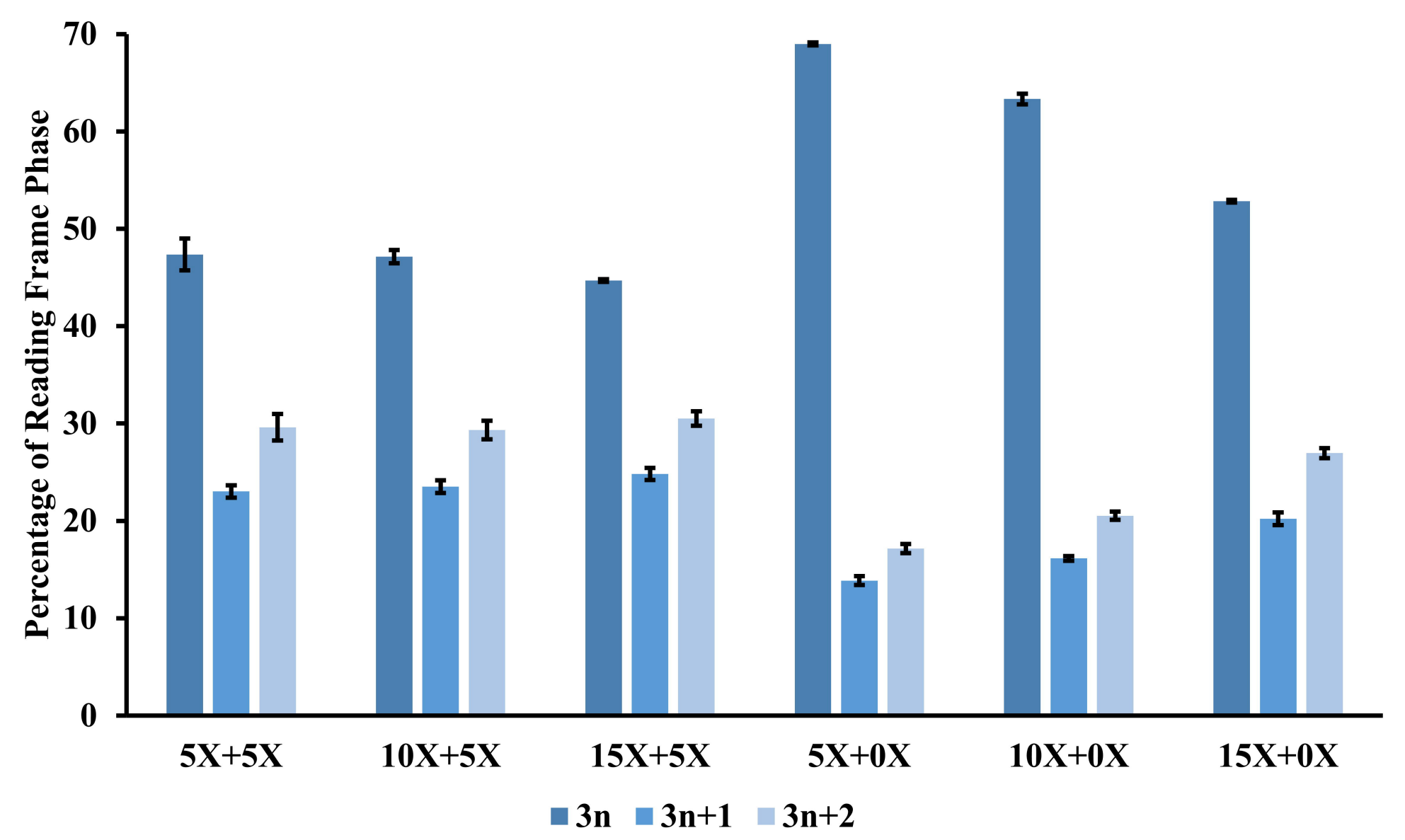

Figure 2 
A

3-mer

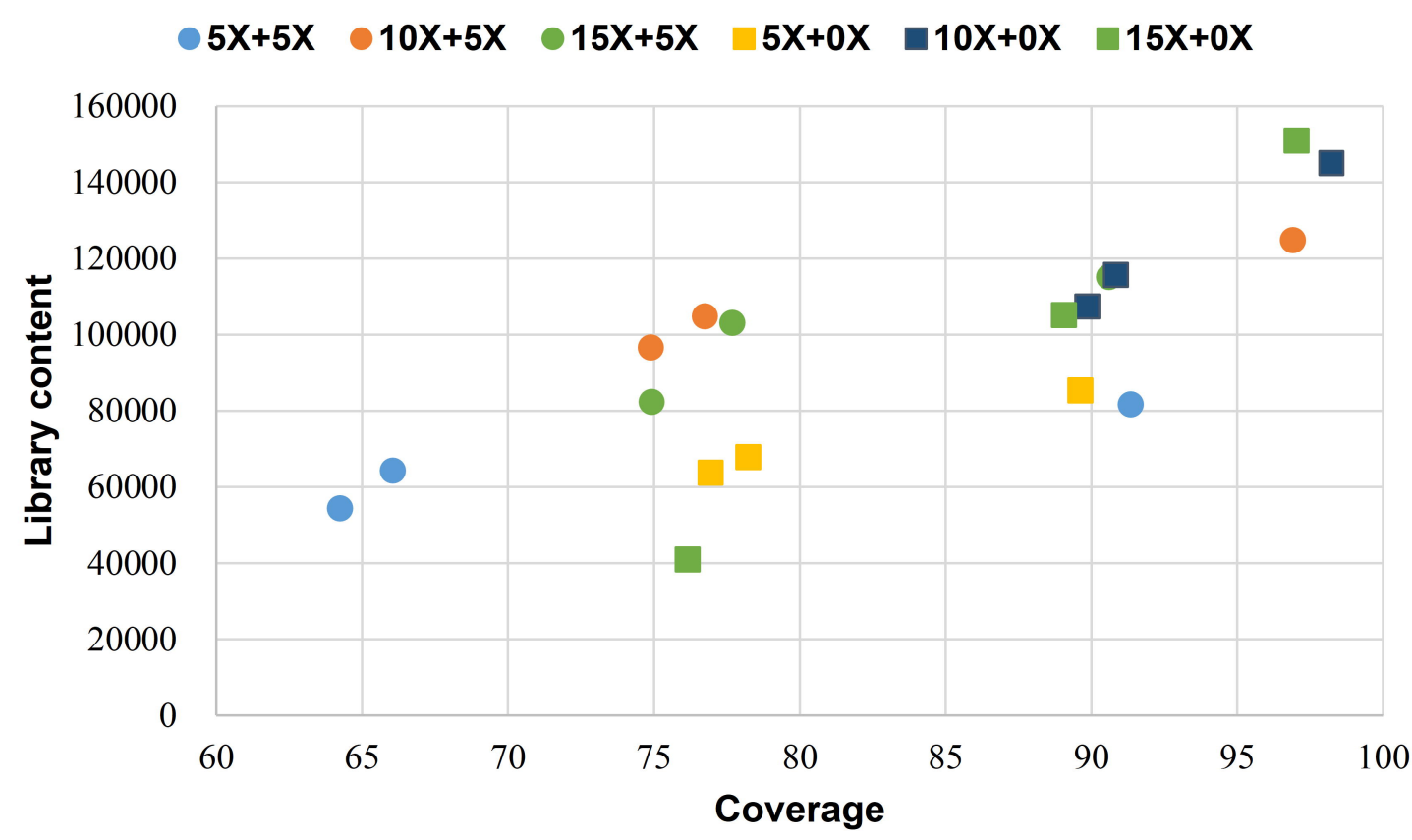

B

4-mer

$5 X+5 X \quad 10 X+5 X \quad 15 X+5 X \quad 5 X+0 X \quad \square 10 X+0 X \quad \square 15 X+0 X$

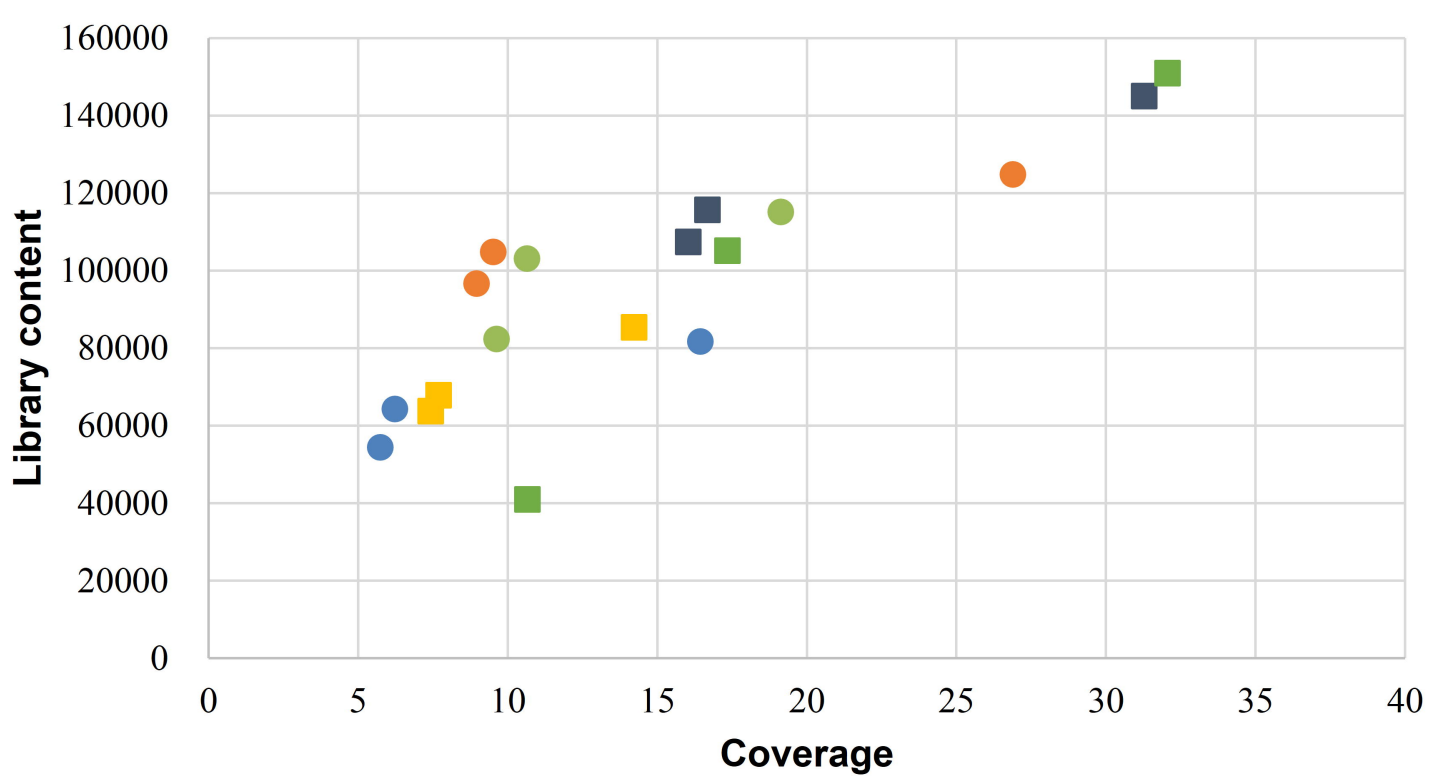

Figure 4 


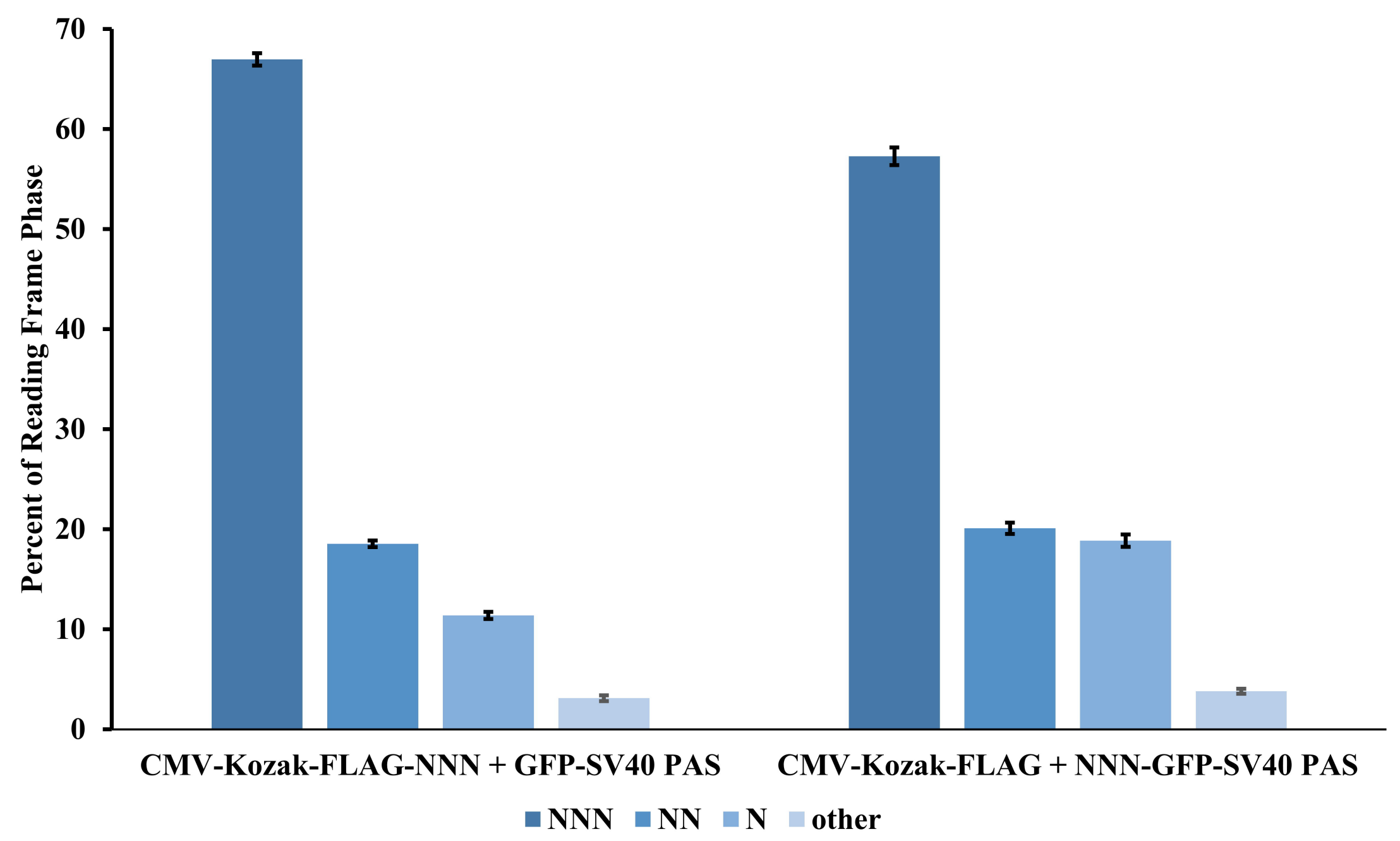

Figure 5 
IdsDNAs

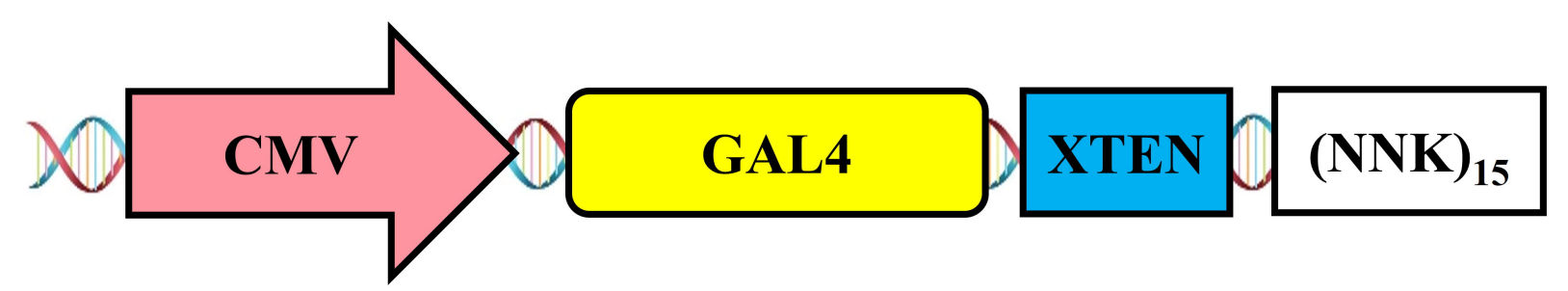

polyA signal

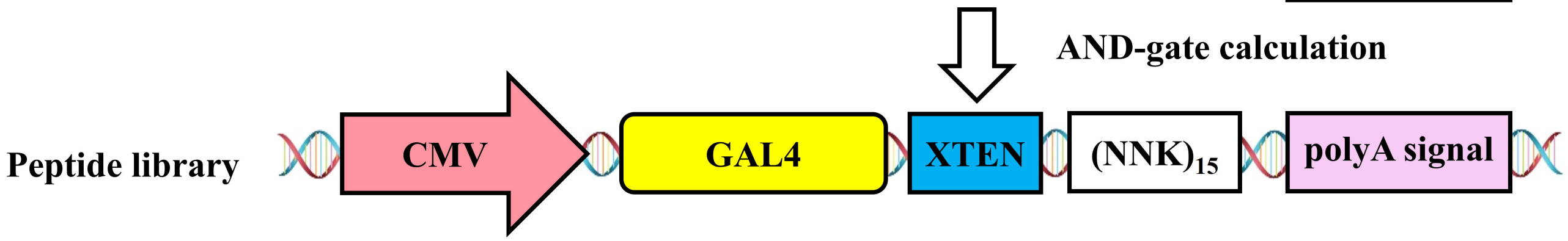

B

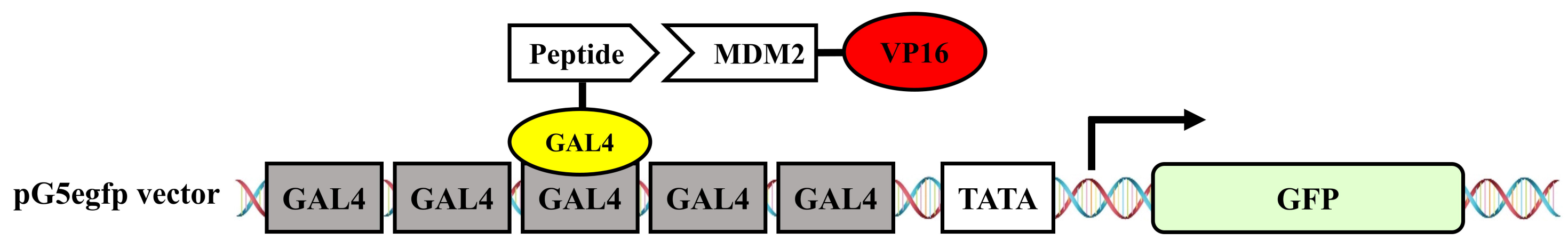


Table 1. High-throughput sequencing (HTS) statistics

\begin{tabular}{|c|c|c|c|}
\hline Library & Description & Clean Read count & Read count in analysis \\
\hline $5 X+5 X(1)$ & \multirow{3}{*}{$\begin{array}{c}\text { Peptide library generated by ldsDNA } \\
\text { CMV-Kozak- }<\mathrm{NNK}\rangle_{5} \text { and }\langle\mathrm{NNK}\rangle_{5} \text {-GFP-SV40 PAS }\end{array}$} & 79179 & 32698 \\
\hline $5 X+5 X(2)$ & & 75950 & 25194 \\
\hline $5 X+5 X(3)$ & & 61489 & 23021 \\
\hline $10 X+5 X(1)$ & \multirow{3}{*}{$\begin{array}{l}\text { Peptide library generated by ldsDNA } \\
\text { ozak- }\langle\mathrm{NNK}\rangle_{10} \text { and }\langle\mathrm{NNK}\rangle_{5}-\mathrm{GFP}-\mathrm{SV} 40 \text { PAS }\end{array}$} & 84191 & 38218 \\
\hline $10 X+5 X(2)$ & & 75195 & 30324 \\
\hline $10 X+5 X(3)$ & & 71341 & 32768 \\
\hline $15 X+5 X(1)$ & \multirow{3}{*}{$\begin{array}{c}\text { Peptide library generated by ldsDNA } \\
\text { MV-Kozak- }\langle\text { NNK }\rangle_{15} \text { and }\langle\text { NNK }\rangle_{5} \text {-GFP-SV40 PAS }\end{array}$} & 67121 & 32273 \\
\hline $15 X+5 X(2)$ & & 39153 & 24083 \\
\hline $15 X+5 X(3)$ & & 82312 & 31784 \\
\hline $5 X+0 X(1)$ & \multirow{3}{*}{$\begin{array}{c}\text { Peptide library generated by ldsDNA } \\
\text { MV-Kozak- }\langle\text { NNK }\rangle_{5} \text { and }\langle\text { NNK }\rangle_{0} \text {-GFP-SV40 PAS }\end{array}$} & 87718 & 30612 \\
\hline $5 X+0 X(2)$ & & 83744 & 22487 \\
\hline $5 X+0 X(3)$ & & 81241 & 23620 \\
\hline $10 X+0 X(1)$ & \multirow{3}{*}{$\begin{array}{c}\text { Peptide library generated by ldsDNA } \\
\text { CMV-Kozak- }\langle\mathrm{NNK}\rangle_{10} \text { and }\langle\mathrm{NNK}\rangle_{0} \text {-GFP-SV40 PAS }\end{array}$} & 82228 & 33130 \\
\hline $10 X+0 X(2)$ & & 74850 & 25512 \\
\hline $10 X+0 X(3)$ & & 86618 & 26676 \\
\hline $15 X+0 X(1)$ & \multirow{3}{*}{$\begin{array}{c}\text { Peptide library generated by ldsDNA } \\
\text { CMV-Kozak- }\langle\text { NNK }\rangle_{15} \text { and }\langle\text { NNK }\rangle_{0} \text {-GFP-SV } 40 \text { PAS }\end{array}$} & 85127 & 33192 \\
\hline $15 X+0 X(2)$ & & 30998 & 9570 \\
\hline $15 X+0 X(3)$ & & 75915 & 23598 \\
\hline
\end{tabular}

Pair-end high-throughput sequencing statistics for all libraries referenced in this study. Sequences formed by ldsDNA-based AND-gate calculation are subject for further analysis. 
Table 2. Percentage of each amino acid of different peptide library

\begin{tabular}{|c|c|c|c|c|c|c|c|}
\hline Amino acid & NNK & $5 X+5 X$ & $10 X+5 X$ & $15 X+5 X$ & $5 \mathrm{X}+0 \mathrm{X}$ & $10 \mathrm{X}+0 \mathrm{X}$ & $15 X+0 X$ \\
\hline Stop codon & 3.125 & $2.34 \pm 0.11$ & $2.46 \pm 0.14$ & $2.28 \pm 0.15$ & $2.59 \pm 0.14$ & $2.47 \pm 0.09$ & $2.31 \pm 0.13$ \\
\hline $\mathrm{A}(\mathrm{Ala})$ & 6.25 & $6.16 \pm 0.08$ & $6.34 \pm 0.18$ & $7.11 \pm 0.19$ & $5.43 \pm 0.12$ & $6.22 \pm 0.13$ & $7.32 \pm 0.26$ \\
\hline C (Cys) & 3.125 & $2.80 \pm 0.10$ & $2.72 \pm 0.09$ & $2.74 \pm 0.24$ & $2.06 \pm 0.17$ & $2.35 \pm 0.03$ & $2.16 \pm 0.09$ \\
\hline $\mathrm{D}(\mathrm{Asp})$ & 3.125 & $2.84 \pm 0.18$ & $2.96 \pm 0.26$ & $2.76 \pm 0.07$ & $3.72 \pm 0.07$ & $3.13 \pm 0.07$ & $2.85 \pm 0.05$ \\
\hline E (Glu) & 3.125 & $2.74 \pm 0.07$ & $2.75 \pm 0.05$ & $2.63 \pm 0.11$ & $3.67 \pm 0.11$ & $3.24 \pm 0.12$ & $3.19 \pm 0.10$ \\
\hline $\mathrm{F}$ (Phe) & 3.125 & $3.34 \pm 0.18$ & $3.34 \pm 0.08$ & $2.88 \pm 0.08$ & $1.71 \pm 0.05$ & $2.52 \pm 0.12$ & $1.88 \pm 0.04$ \\
\hline G (Gly) & 6.25 & $5.23 \pm 0.23$ & $5.28 \pm 0.14$ & $5.85 \pm 0.08$ & $4.35 \pm 0.07$ & $4.89 \pm 0.13$ & $5.68 \pm 0.14$ \\
\hline H (His) & 3.125 & $3.54 \pm 0.17$ & $3.41 \pm 0.18$ & $3.26 \pm 0.20$ & $4.35 \pm 0.11$ & $3.72 \pm 0.14$ & $3.21 \pm 0.10$ \\
\hline I (Ile) & 3.125 & $3.45 \pm 0.08$ & $3.40 \pm 0.38$ & $2.76 \pm 0.12$ & $3.49 \pm 0.26$ & $3.05 \pm 0.12$ & $2.48 \pm 0.07$ \\
\hline K (Lys) & 3.125 & $3.57 \pm 0.16$ & $3.20 \pm 0.06$ & $2.70 \pm 0.07$ & $5.93 \pm 0.17$ & $4.08 \pm 0.09$ & $3.32 \pm 0.15$ \\
\hline L (Leu) & 9.375 & $8.84 \pm 0.16$ & $9.35 \pm 0.17$ & $8.98 \pm 0.28$ & $6.69 \pm 0.22$ & $8.63 \pm 0.11$ & $7.93 \pm 0.12$ \\
\hline M (Met) & 3.125 & $2.91 \pm 0.09$ & $2.90 \pm 0.18$ & $2.68 \pm 0.03$ & $3.54 \pm 0.10$ & $3.04 \pm 0.04$ & $2.59 \pm 0.08$ \\
\hline N (Asn) & 3.125 & $4.23 \pm 0.18$ & $3.55 \pm 0.16$ & $2.90 \pm 0.07$ & $6.33 \pm 0.09$ & $4.37 \pm 0.12$ & $3.35 \pm 0.12$ \\
\hline P (Pro) & 6.25 & $8.16 \pm 0.15$ & $9.20 \pm 0.52$ & $10.40 \pm 0.28$ & $7.34 \pm 0.10$ & $9.41 \pm 0.16$ & $11.32 \pm 0.11$ \\
\hline $\mathrm{Q}(\mathrm{Gln})$ & 3.125 & $3.13 \pm 0.11$ & $3.12 \pm 0.04$ & $3.17 \pm 0.09$ & $4.13 \pm 0.11$ & $3.89 \pm 0.02$ & $3.82 \pm 0.09$ \\
\hline R (Arg) & 9.375 & $8.34 \pm 0.28$ & $8.47 \pm 0.24$ & $9.79 \pm 0.23$ & $8.74 \pm 0.08$ & $9.18 \pm 0.25$ & $10.68 \pm 0.29$ \\
\hline $\mathrm{S}($ Ser $)$ & 9.375 & $10.30 \pm 0.16$ & $9.76 \pm 0.30$ & $9.68 \pm 0.20$ & $8.54 \pm 0.14$ & $8.63 \pm 0.17$ & $8.90 \pm 0.32$ \\
\hline $\mathrm{T}$ (Thr) & 6.25 & $7.24 \pm 0.17$ & $6.64 \pm 0.27$ & $6.94 \pm 0.12$ & $8.88 \pm 0.21$ & $7.27 \pm 0.17$ & $7.58 \pm 0.15$ \\
\hline V (Val) & 6.25 & $5.66 \pm 0.24$ & $5.56 \pm 0.19$ & $5.31 \pm 0.21$ & $3.59 \pm 0.11$ & $4.58 \pm 0.07$ & $4.51 \pm 0.21$ \\
\hline W (Trp) & 3.125 & $2.30 \pm 0.06$ & $2.70 \pm 0.31$ & $2.60 \pm 0.04$ & $1.96 \pm 0.07$ & $2.33 \pm 0.05$ & $2.36 \pm 0.07$ \\
\hline Y (Tyr) & 3.125 & $2.89 \pm 0.11$ & $2.89 \pm 0.21$ & $2.59 \pm 0.07$ & $2.96 \pm 0.11$ & $2.99 \pm 0.14$ & $2.57 \pm 0.16$ \\
\hline
\end{tabular}

In-frame (3n) nucleotide sequences generated by ldsDNA-based AND-gate are translated into polypeptide sequences. The total number of each amino acid in each 
library is summarized. The average percentage of different amino acid is presented. All data are displayed as the mean $\pm \mathrm{SD}, \mathrm{n}=3$. The theoretical amino acid percentage of NNK degenerate codon is also presented. 
Supplementary Table 1. List of PCR primers used in study.

\begin{tabular}{|c|c|c|}
\hline Amplicon & Forward primer & Reverse primer \\
\hline CMV-Kozak- $<$ NNK $>_{5}$ & $\begin{array}{l}\text { CTTTCCTGCGTTATCCCCT } \\
\text { GATTCT }\end{array}$ & $\begin{array}{l}\text { MNNMNNMNNMNNMNNCAC } \\
\text { CATGGTGGCGACCGGTAGC } \\
\text { GCT }\end{array}$ \\
\hline CMV-Kozak- $\langle\text { NNK }\rangle_{10}$ & $\begin{array}{l}\text { CTTTCCTGCGTTATCCCCT } \\
\text { GATTCT }\end{array}$ & $\begin{array}{l}\text { MNNMNNMNNMNNMNNMN } \\
\text { NMNNMNNMNNMNNCACCA } \\
\text { TGGTGGCGACCGGTAGCGCT }\end{array}$ \\
\hline CMV-Kozak- $\langle\text { NNK }\rangle_{15}$ & $\begin{array}{l}\text { CTTTCCTGCGTTATCCCCT } \\
\text { GATTCT }\end{array}$ & $\begin{array}{l}\text { MNNMNNMNNMNNMNNMN } \\
\text { NMNNMNNMNNMNNMNNM } \\
\text { NNMNNMNNMNNCACCATG } \\
\text { GTGGCGACCGGTAGCGCT }\end{array}$ \\
\hline$\langle\mathrm{NNK}\rangle_{5}$-GFP-SV40 PAS & $\begin{array}{l}\text { NNKNNKNNKNNKNNKAT } \\
\text { GGTGAGCAAGGGCGAGG } \\
\text { AGCTGT }\end{array}$ & $\begin{array}{l}\text { CGGCCTATTGGTTAAAAAAT } \\
\text { GAGCT }\end{array}$ \\
\hline$\langle\mathrm{NNK}\rangle_{0}$-GFP-SV40 PAS & $\begin{array}{l}\text { ATGGTGAGCAAGGGCGA } \\
\text { GGAGCTGT }\end{array}$ & $\begin{array}{l}\text { CGGCCTATTGGTTAAAAAAT } \\
\text { GAGCT }\end{array}$ \\
\hline CMV-Kozak-FLAG-NNN & $\begin{array}{l}\text { CTTTCCTGCGTTATCCCCT } \\
\text { GATTCT }\end{array}$ & $\begin{array}{l}\text { NNNTTTATCGTCATCGTCTT } \\
\text { TGTAGTCCACCATGGTGGCG } \\
\text { ACCGGTAGCGCT }\end{array}$ \\
\hline GFP-SV40 PAS & $\begin{array}{l}\text { A*TGGTGAGCAAGGGCGA } \\
\text { GGAGCTGT }\end{array}$ & $\begin{array}{l}\text { CGGCCTATTGGTTAAAAAAT } \\
\text { GAGCT }\end{array}$ \\
\hline CMV-Kozak-FLAG & $\begin{array}{l}\text { CTTTCCTGCGTTATCCCCT } \\
\text { GATTCT }\end{array}$ & $\begin{array}{l}\text { T*TTATCGTCATCGTCTTTGT } \\
\text { AGTCCACCATGGTGGCGACC } \\
\text { GGTAGCGCT }\end{array}$ \\
\hline NNN-GFP-SV40 & $\begin{array}{l}\text { NNNATGGTGAGCAAGGG } \\
\text { CGAGGAGCTGT }\end{array}$ & $\begin{array}{l}\text { CGGCCTATTGGTTAAAAAAT } \\
\text { GAGCT }\end{array}$ \\
\hline $\begin{array}{l}\text { Amplicon for PE-sequencing } \\
\text { library construction }\end{array}$ & $\begin{array}{l}\text { [Barcode]TCAGATCCGCTA } \\
\text { GCGCTACC }\end{array}$ & $\begin{array}{l}\text { [Barcode]GAACTTCAGGGTCA } \\
\text { GCTTGC }\end{array}$ \\
\hline $\begin{array}{l}\text { CMV-GAL4-XTEN-<NNK }> \\
15\end{array}$ & $\begin{array}{l}\text { TACGGTTCCTGGCCTTTT } \\
\text { GCTGGCC }\end{array}$ & $\begin{array}{l}\text { MNNMNNMNNMNNMNNMN } \\
\text { NMNNMNNMNNMNNMNNM } \\
\text { NNMNNMNNMNNGGATTCG } \\
\text { GGTGTGGCGGACTCTGAG }\end{array}$ \\
\hline SV40 PAS & $\begin{array}{l}\text { CTCTAGATAACTGAGGTA } \\
\text { CCTGAATAACTAAGGCCG } \\
\text { CTT }\end{array}$ & $\begin{array}{l}\text { ATACCGCATCAGGAAATTGT } \\
\text { AAGCG }\end{array}$ \\
\hline
\end{tabular}




\begin{tabular}{l|l|l}
\hline $\begin{array}{l}\text { Amplicon for PE-sequencing } \\
\text { library construction (MDM2 } \\
\text { interacting peptide library) }\end{array}$ & [Barcode]GGTCAAAGACAG & $\begin{array}{l}\text { [Barcode]ACCCTCACTAAAGG } \\
\text { GAAGCG }\end{array}$ \\
\hline
\end{tabular}

$\mathrm{N}=\mathrm{T}$ or $\mathrm{C}$ or $\mathrm{A}$ or $\mathrm{G} ; \mathrm{K}=\mathrm{T}$ or $\mathrm{G} ; \mathrm{M}=\mathrm{C}$ or $\mathrm{A} ; *=$ phosphorothioate bond. 
Supplementary Table 2. Percentage of each nucleotide at each position of peptide library codons

\begin{tabular}{|c|c|c|c|c|c|c|c|}
\hline Codon position & Nucleotide & $5 X+5 X$ & $10 X+5 X$ & $15 X+5 X$ & $5 X+0 X$ & $10 \mathrm{X}+0 \mathrm{X}$ & $15 \mathrm{X}+0 \mathrm{X}$ \\
\hline \multirow[b]{4}{*}{ First } & $\mathrm{T}$ & $23.69 \pm 0.30$ & $24.31 \pm 0.45$ & $22.86 \pm 0.35$ & $17.96 \pm 0.45$ & $21.21 \pm 0.21$ & $19.67 \pm 0.36$ \\
\hline & $\mathrm{C}$ & $26.43 \pm 0.30$ & $28.23 \pm 0.59$ & $30.39 \pm 0.29$ & $25.64 \pm 0.34$ & $29.42 \pm 0.28$ & $31.93 \pm 0.41$ \\
\hline & A & $27.26 \pm 0.23$ & $24.58 \pm 0.35$ & $23.09 \pm 0.13$ & $35.64 \pm 0.22$ & $27.31 \pm 0.27$ & $24.84 \pm 0.15$ \\
\hline & $\mathrm{G}$ & $22.63 \pm 0.18$ & $22.89 \pm 0.30$ & $23.66 \pm 0.24$ & $20.75 \pm 0.11$ & $22.06 \pm 0.24$ & $23.55 \pm 0.42$ \\
\hline \multirow[b]{4}{*}{ Second } & $\mathrm{T}$ & $24.20 \pm 0.40$ & $24.55 \pm 0.67$ & $22.60 \pm 0.10$ & $19.01 \pm 0.02$ & $21.82 \pm 0.24$ & $19.39 \pm 0.35$ \\
\hline & $\mathrm{C}$ & $28.78 \pm 0.19$ & $29.43 \pm 0.80$ & $31.66 \pm 0.35$ & $26.53 \pm 0.31$ & $28.91 \pm 0.45$ & $32.50 \pm 0.29$ \\
\hline & A & $25.23 \pm 0.38$ & $24.29 \pm 0.39$ & $22.19 \pm 0.34$ & $33.66 \pm 0.07$ & $27.87 \pm 0.39$ & $24.55 \pm 0.50$ \\
\hline & $\mathrm{G}$ & $21.79 \pm 0.29$ & $21.74 \pm 0.10$ & $23.55 \pm 0.09$ & $20.80 \pm 0.35$ & $21.40 \pm 0.33$ & $23.55 \pm 0.13$ \\
\hline \multirow[b]{4}{*}{ Third } & $\mathrm{T}$ & $52.50 \pm 0.42$ & $51.13 \pm 0.24$ & $47.80 \pm 0.04$ & $49.64 \pm 0.74$ & $48.56 \pm 0.34$ & $45.07 \pm 0.44$ \\
\hline & $\mathrm{C}$ & $0.65 \pm 0.04$ & $0.66 \pm 0.06$ & $1.51 \pm 0.03$ & $0.48 \pm 0.05$ & $0.31 \pm 0.03$ & $0.83 \pm 0.08$ \\
\hline & A & $0.86 \pm 0.10$ & $0.61 \pm 0.04$ & $1.03 \pm 0.08$ & $0.42 \pm 0.03$ & $0.36 \pm 0.04$ & $0.60 \pm 0.05$ \\
\hline & $\mathrm{G}$ & $45.98 \pm 0.31$ & $47.60 \pm 0.29$ & $49.66 \pm 0.12$ & $49.46 \pm 0.71$ & $50.77 \pm 0.33$ & $53.50 \pm 0.33$ \\
\hline
\end{tabular}

The total number of each nucleotide at each position of peptide library codons is summarized. The average percentage of different nucleotide at each codon base is presented. All data are displayed as the mean $\pm \mathrm{SD}, \mathrm{n}=3$. 
Supplementary Table 3. Percentage of triple-nucleotides formed by LBAG calculation

\begin{tabular}{|c|c|c|}
\hline Triple nucleotides & $\begin{array}{l}\text { CMV-Kozak-FLAG-NNN + } \\
\text { GFP-SV40 PAS }\end{array}$ & $\begin{array}{l}\text { CMV-Kozak-FLAG } \\
\text { NNN-GFP-SV40 PAS }\end{array}$ \\
\hline AAA & $2.00 \pm 0.21$ & $1.93 \pm 0.52$ \\
\hline AAC & $3.29 \pm 0.11$ & $4.82 \pm 0.31$ \\
\hline AAG & $2.22 \pm 0.23$ & $0.28 \pm 0.06$ \\
\hline AAT & $2.92 \pm 0.20$ & $2.78 \pm 0.11$ \\
\hline $\mathrm{ACA}$ & $2.18 \pm 0.33$ & $1.19 \pm 0.26$ \\
\hline ACC & $3.74 \pm 0.58$ & $2.14 \pm 0.21$ \\
\hline ACG & $1.68 \pm 0.12$ & $0.13 \pm 0.11$ \\
\hline ACT & $2.53 \pm 0.09$ & $1.31 \pm 0.18$ \\
\hline AGA & $0.85 \pm 0.10$ & $0.59 \pm 0.12$ \\
\hline AGC & $1.48 \pm 0.16$ & $1.58 \pm 0.20$ \\
\hline AGG & $0.76 \pm 0.14$ & $0.07 \pm 0.01$ \\
\hline AGT & $0.68 \pm 0.12$ & $0.88 \pm 0.25$ \\
\hline ATA & $1.77 \pm 0.18$ & $1.76 \pm 0.32$ \\
\hline ATC & $3.15 \pm 0.17$ & $5.06 \pm 0.63$ \\
\hline ATG & $1.86 \pm 0.15$ & $0.21 \pm 0.04$ \\
\hline ATT & $2.88 \pm 0.19$ & $2.27 \pm 0.24$ \\
\hline CAA & $1.84 \pm 0.30$ & $2.52 \pm 0.24$ \\
\hline CAC & $2.45 \pm 0.37$ & $4.58 \pm 0.77$ \\
\hline CAG & $1.54 \pm 0.34$ & $0.27 \pm 0.11$ \\
\hline CAT & $2.33 \pm 0.37$ & $3.37 \pm 0.96$ \\
\hline $\mathrm{CCA}$ & $1.53 \pm 0.33$ & $2.13 \pm 0.14$ \\
\hline $\mathrm{CCC}$ & $1.89 \pm 0.09$ & $4.29 \pm 0.10$ \\
\hline CCG & $1.17 \pm 0.26$ & $0.29 \pm 0.07$ \\
\hline $\mathrm{CCT}$ & $2.11 \pm 0.21$ & $2.18 \pm 0.39$ \\
\hline CGA & $0.79 \pm 0.07$ & $0.53 \pm 0.17$ \\
\hline CGC & $0.93 \pm 0.13$ & $1.08 \pm 0.25$ \\
\hline CGG & $0.53 \pm 0.04$ & $0.12 \pm 0.06$ \\
\hline CGT & $0.60 \pm 0.05$ & $0.74 \pm 0.30$ \\
\hline CTA & $1.50 \pm 0.32$ & $2.48 \pm 0.25$ \\
\hline CTC & $2.11 \pm 0.26$ & $5.69 \pm 0.43$ \\
\hline CTG & $1.34 \pm 0.25$ & $0.48 \pm 0.22$ \\
\hline CTT & $1.93 \pm 0.14$ & $2.94 \pm 0.25$ \\
\hline GAA & $1.19 \pm 0.22$ & $0.48 \pm 0.13$ \\
\hline GAC & $1.81 \pm 0.36$ & $1.15 \pm 0.24$ \\
\hline GAG & $1.18 \pm 0.06$ & $0.07 \pm 0.02$ \\
\hline GAT & $1.58 \pm 0.17$ & $0.75 \pm 0.22$ \\
\hline GCA & $1.24 \pm 0.24$ & $0.40 \pm 0.04$ \\
\hline GCC & $2.00 \pm 0.20$ & $0.92 \pm 0.16$ \\
\hline GCG & $0.92 \pm 0.10$ & $0.11 \pm 0.09$ \\
\hline GCT & $1.68 \pm 0.32$ & $0.50 \pm 0.07$ \\
\hline
\end{tabular}




\begin{tabular}{|c|c|c|}
\hline GGA & $0.38 \pm 0.09$ & $0.20 \pm 0.03$ \\
\hline GGC & $0.65 \pm 0.15$ & $0.47 \pm 0.10$ \\
\hline GGG & $0.29 \pm 0.06$ & $0.02 \pm 0.02$ \\
\hline GGT & $0.33 \pm 0.07$ & $0.20 \pm 0.03$ \\
\hline GTA & $0.80 \pm 0.19$ & $0.58 \pm 0.20$ \\
\hline GTC & $1.35 \pm 0.47$ & $0.92 \pm 0.17$ \\
\hline GTG & $0.78 \pm 0.11$ & $0.10 \pm 0.09$ \\
\hline GTT & $1.25 \pm 0.04$ & $0.51 \pm 0.02$ \\
\hline TAA & $1.69 \pm 0.14$ & $2.12 \pm 0.21$ \\
\hline TAC & $2.65 \pm 0.49$ & $3.70 \pm 0.25$ \\
\hline TAG & $1.24 \pm 0.13$ & $0.32 \pm 0.08$ \\
\hline TAT & $1.95 \pm 0.27$ & $3.04 \pm 0.25$ \\
\hline $\mathrm{TCA}$ & $1.51 \pm 0.38$ & $2.38 \pm 0.25$ \\
\hline $\mathrm{TCC}$ & $2.47 \pm 0.13$ & $3.97 \pm 0.17$ \\
\hline $\mathrm{TCG}$ & $1.45 \pm 0.22$ & $0.23 \pm 0.07$ \\
\hline $\mathrm{TCT}$ & $1.86 \pm 0.18$ & $2.48 \pm 0.24$ \\
\hline TGA & $0.74 \pm 0.18$ & $0.96 \pm 0.12$ \\
\hline TGC & $1.03 \pm 0.03$ & $1.60 \pm 0.25$ \\
\hline TGG & $0.62 \pm 0.15$ & $0.16 \pm 0.06$ \\
\hline TGT & $0.57 \pm 0.06$ & $1.11 \pm 0.30$ \\
\hline TTA & $1.03 \pm 0.14$ & $1.86 \pm 0.36$ \\
\hline TTC & $1.99 \pm 0.26$ & $4.85 \pm 0.44$ \\
\hline TTG & $1.37 \pm 0.05$ & $0.33 \pm 0.24$ \\
\hline TTT & $1.85 \pm 0.15$ & $2.82 \pm 0.28$ \\
\hline
\end{tabular}

The total number of each triple-nucleotides formed by LBAG calculation is summarized. The average percentage of different triple-nucleotides is presented. All data are displayed as the mean $\pm \mathrm{SD}, \mathrm{n}=4$. 
Supplementary Table 4. Potential MDM2 interacting peptide

\begin{tabular}{|c|c|c|}
\hline No & Peptide sequence & Read counts \\
\hline 1 & CAGM I KQSQLRHPKS & 47 \\
\hline 2 & KLR I MMQAFRHVYWN & 39 \\
\hline 3 & DRQT N Y G N L P ARDV & 25 \\
\hline 4 & $S \underline{F} V \mid R \underline{W} T A \underline{L}$ WP GPR S & 23 \\
\hline 5 & MYSKWDPMRHDF SET & 21 \\
\hline 6 & $H S L D L Q N Q \mid Q T C E E W$ & 20 \\
\hline 7 & S TPWLAMMLR L GP F N & 17 \\
\hline 8 & N S C R L M L H P P MRP L & 16 \\
\hline 9 & P T SREFCNA I HACLV & 14 \\
\hline 10 & K Y F CMPETRMHPRR T & 14 \\
\hline
\end{tabular}

High-throughput sequencing decoded top ten hit amino acid sequences in GFP positive cells obtained from the LBAG mammalian two-hybrid system. Three crucial residues (Phe, Trp and Leu) mediating MDM2-p53 interaction are shown in underline bold. 


\section{Supplementary materials}

ldsDNA sequences in study:

$>\mathrm{CMV}-\mathrm{Kozak}-\langle\mathrm{NNK}\rangle_{5}$

CTTTCCTGCGTTATCCCCTGATTCTGTGGATAACCGTATTACCGCCATGCATTAGT TATTAATAGTAATCAATTACGGGGTCATTAGTTCATAGCCCATATATGGAGTTCC GCGTTACATAACTTACGGTAAATGGCCCGCCTGGCTGACCGCCCAACGACCCCCG CCCATTGACGTCAATAATGACGTATGTTCCCATAGTAACGCCAATAGGGACTTTC CATTGACGTCAATGGGTGGAGTATTTACGGTAAACTGCCCACTTGGCAGTACATC AAGTGTATCATATGCCAAGTACGCCCCCTATTGACGTCAATGACGGTAAATGGCC CGCCTGGCATTATGCCCAGTACATGACCTTATGGGACTTTCCTACTTGGCAGTAC ATCTACGTATTAGTCATCGCTATTACCATGGTGATGCGGTTTTGGCAGTACATCAA TGGGCGTGGATAGCGGTTTGACTCACGGGGATTTCCAAGTCTCCACCCCATTGAC GTCAATGGGAGTTTGTTTTGGCACCAAAATCAACGGGACTTTCCAAAATGTCGTA ACAACTCCGCCCCATTGACGCAAATGGGCGGTAGGCGTGTACGGTGGGAGGTCT ATATAAGCAGAGCTGGTTTAGTGAACCGTCAGATCCGCTAGCGCTACCGGTCGCC ACCATGGTGNNKNNKNNKNNKNNK

$>\mathrm{CMV}-$ Kozak- $\langle\mathrm{NNK}\rangle_{10}$

CTTTCCTGCGTTATCCCCTGATTCTGTGGATAACCGTATTACCGCCATGCATTAGT TATTAATAGTAATCAATTACGGGGTCATTAGTTCATAGCCCATATATGGAGTTCC GCGTTACATAACTTACGGTAAATGGCCCGCCTGGCTGACCGCCCAACGACCCCCG CCCATTGACGTCAATAATGACGTATGTTCCCATAGTAACGCCAATAGGGACTTTC CATTGACGTCAATGGGTGGAGTATTTACGGTAAACTGCCCACTTGGCAGTACATC AAGTGTATCATATGCCAAGTACGCCCCCTATTGACGTCAATGACGGTAAATGGCC CGCCTGGCATTATGCCCAGTACATGACCTTATGGGACTTTCCTACTTGGCAGTAC ATCTACGTATTAGTCATCGCTATTACCATGGTGATGCGGTTTTGGCAGTACATCAA TGGGCGTGGATAGCGGTTTGACTCACGGGGATTTCCAAGTCTCCACCCCATTGAC GTCAATGGGAGTTTGTTTTGGCACCAAAATCAACGGGACTTTCCAAAATGTCGTA ACAACTCCGCCCCATTGACGCAAATGGGCGGTAGGCGTGTACGGTGGGAGGTCT ATATAAGCAGAGCTGGTTTAGTGAACCGTCAGATCCGCTAGCGCTACCGGTCGCC ACCATGGTGNNKNNKNNKNNKNNKNNKNNKNNKNNKNNK

$>\mathrm{CMV}-$ Kozak- $\langle\mathrm{NNK}\rangle_{15}$

CTTTCCTGCGTTATCCCCTGATTCTGTGGATAACCGTATTACCGCCATGCATTAGT TATTAATAGTAATCAATTACGGGGTCATTAGTTCATAGCCCATATATGGAGTTCC GCGTTACATAACTTACGGTAAATGGCCCGCCTGGCTGACCGCCCAACGACCCCCG CCCATTGACGTCAATAATGACGTATGTTCCCATAGTAACGCCAATAGGGACTTTC CATTGACGTCAATGGGTGGAGTATTTACGGTAAACTGCCCACTTGGCAGTACATC AAGTGTATCATATGCCAAGTACGCCCCCTATTGACGTCAATGACGGTAAATGGCC CGCCTGGCATTATGCCCAGTACATGACCTTATGGGACTTTCCTACTTGGCAGTAC 
ATCTACGTATTAGTCATCGCTATTACCATGGTGATGCGGTTTTGGCAGTACATCAA TGGGCGTGGATAGCGGTTTGACTCACGGGGATTTCCAAGTCTCCACCCCATTGAC GTCAATGGGAGTTTGTTTTGGCACCAAAATCAACGGGACTTTCCAAAATGTCGTA ACAACTCCGCCCCATTGACGCAAATGGGCGGTAGGCGTGTACGGTGGGAGGTCT ATATAAGCAGAGCTGGTTTAGTGAACCGTCAGATCCGCTAGCGCTACCGGTCGCC ACCATGGTGNNKNNKNNKNNKNNKNNKNNKNNKNNKNNKNNKNNKNNKNNKN NK

$>\left\langle\mathrm{NNK}>_{5}\right.$-GFP-SV40 PAS

NNKNNKNNKNNKNNKATGGTGAGCAAGGGCGAGGAGCTGTTCACCGGGGTGGT GCCCATCCTGGTCGAGCTGGACGGCGACGTAAACGGCCACAAGTTCAGCGTGTCC GGCGAGGGCGAGGGCGATGCCACCTACGGCAAGCTGACCCTGAAGTTCATCTGC ACCACCGGCAAGCTGCCCGTGCCCTGGCCCACCCTCGTGACCACCCTGACCTACG GCGTGCAGTGCTTCAGCCGCTACCCCGACCACATGAAGCAGCACGACTTCTTCAA GTCCGCCATGCCCGAAGGCTACGTCCAGGAGCGCACCATCTTCTTCAAGGACGAC GGCAACTACAAGACCCGCGCCGAGGTGAAGTTCGAGGGCGACACCCTGGTGAAC CGCATCGAGCTGAAGGGCATCGACTTCAAGGAGGACGGCAACATCCTGGGGCAC AAGCTGGAGTACAACTACAACAGCCACAACGTCTATATCATGGCCGACAAGCAG AAGAACGGCATCAAGGTGAACTTCAAGATCCGCCACAACATCGAGGACGGCAGC GTGCAGCTCGCCGACCACTACCAGCAGAACACCCCCATCGGCGACGGCCCCGTG CTGCTGCCCGACAACCACTACCTGAGCACCCAGTCCGCCCTGAGCAAAGACCCCA ACGAGAAGCGCGATCACATGGTCCTGCTGGAGTTCGTGACCGCCGCCGGGATCA CTCTCGGCATGGACGAGCTGTACAAGTCCGGACTCAGATCTCGAGCTCAAGCTTC GAATTCTGCAGTCGACGGTACCGCGGGCCCGGGATCCACCGGATCTAGATAACTG ATCATAATCAGCCATACCACATTTGTAGAGGTTTTACTTGCTTTAAAAAACCTCCC ACACCTCCCCCTGAACCTGAAACATAAAATGAATGCAATTGTTGTTGTTAACTTG TTTATTGCAGCTTATAATGGTTACAAATAAAGCAATAGCATCACAAATTTCACAA ATAAAGCATTTTTTTCACTGCATTCTAGTTGTGGTTTGTCCAAACTCATCAATGTA TCTTAACGCGTAAATTGTAAGCGTTAATATTTTGTTAAAATTCGCGTTAAATTTTT GTTAAATCAGCTCATTTTTTAACCAATAGGCCG

$><\mathrm{NNK}>_{0}$-GFP-SV40 PAS

ATGGTGAGCAAGGGCGAGGAGCTGTTCACCGGGGTGGTGCCCATCCTGGTCGAG CTGGACGGCGACGTAAACGGCCACAAGTTCAGCGTGTCCGGCGAGGGCGAGGGC GATGCCACCTACGGCAAGCTGACCCTGAAGTTCATCTGCACCACCGGCAAGCTGC CCGTGCCCTGGCCCACCCTCGTGACCACCCTGACCTACGGCGTGCAGTGCTTCAG CCGCTACCCCGACCACATGAAGCAGCACGACTTCTTCAAGTCCGCCATGCCCGAA GGCTACGTCCAGGAGCGCACCATCTTCTTCAAGGACGACGGCAACTACAAGACC CGCGCCGAGGTGAAGTTCGAGGGCGACACCCTGGTGAACCGCATCGAGCTGAAG GGCATCGACTTCAAGGAGGACGGCAACATCCTGGGGCACAAGCTGGAGTACAAC TACAACAGCCACAACGTCTATATCATGGCCGACAAGCAGAAGAACGGCATCAAG 
GTGAACTTCAAGATCCGCCACAACATCGAGGACGGCAGCGTGCAGCTCGCCGAC CACTACCAGCAGAACACCCCCATCGGCGACGGCCCCGTGCTGCTGCCCGACAACC ACTACCTGAGCACCCAGTCCGCCCTGAGCAAAGACCCCAACGAGAAGCGCGATC ACATGGTCCTGCTGGAGTTCGTGACCGCCGCCGGGATCACTCTCGGCATGGACGA GCTGTACAAGTCCGGACTCAGATCTCGAGCTCAAGCTTCGAATTCTGCAGTCGAC GGTACCGCGGGCCCGGGATCCACCGGATCTAGATAACTGATCATAATCAGCCATA CCACATTTGTAGAGGTTTTACTTGCTTTAAAAAACCTCCCACACCTCCCCCTGAAC CTGAAACATAAAATGAATGCAATTGTTGTTGTTAACTTGTTTATTGCAGCTTATAA TGGTTACAAATAAAGCAATAGCATCACAAATTTCACAAATAAAGCATTTTTTTCA CTGCATTCTAGTTGTGGTTTGTCCAAACTCATCAATGTATCTTAACGCGTAAATTG TAAGCGTTAATATTTTGTTAAAATTCGCGTTAAATTTTTGTTAAATCAGCTCATTT TTTAACCAATAGGCCG

>CMV-Kozak-FLAG-NNN

CTTTCCTGCGTTATCCCCTGATTCTGTGGATAACCGTATTACCGCCATGCATTAGT TATTAATAGTAATCAATTACGGGGTCATTAGTTCATAGCCCATATATGGAGTTCC GCGTTACATAACTTACGGTAAATGGCCCGCCTGGCTGACCGCCCAACGACCCCCG CCCATTGACGTCAATAATGACGTATGTTCCCATAGTAACGCCAATAGGGACTTTC CATTGACGTCAATGGGTGGAGTATTTACGGTAAACTGCCCACTTGGCAGTACATC AAGTGTATCATATGCCAAGTACGCCCCCTATTGACGTCAATGACGGTAAATGGCC CGCCTGGCATTATGCCCAGTACATGACCTTATGGGACTTTCCTACTTGGCAGTAC

ATCTACGTATTAGTCATCGCTATTACCATGGTGATGCGGTTTTGGCAGTACATCAA TGGGCGTGGATAGCGGTTTGACTCACGGGGATTTCCAAGTCTCCACCCCATTGAC GTCAATGGGAGTTTGTTTTGGCACCAAAATCAACGGGACTTTCCAAAATGTCGTA ACAACTCCGCCCCATTGACGCAAATGGGCGGTAGGCGTGTACGGTGGGAGGTCT ATATAAGCAGAGCTGGTTTAGTGAACCGTCAGATCCGCTAGCGCTACCGGTCGCC ACCATGGTGGACTACAAAGACGATGACGATAAANNN

> GFP-SV40 PAS

A*TGGTGAGCAAGGGCGAGGAGCTGTTCACCGGGGTGGTGCCCATCCTGGTCGAG CTGGACGGCGACGTAAACGGCCACAAGTTCAGCGTGTCCGGCGAGGGCGAGGGC GATGCCACCTACGGCAAGCTGACCCTGAAGTTCATCTGCACCACCGGCAAGCTGC CCGTGCCCTGGCCCACCCTCGTGACCACCCTGACCTACGGCGTGCAGTGCTTCAG CCGCTACCCCGACCACATGAAGCAGCACGACTTCTTCAAGTCCGCCATGCCCGAA GGCTACGTCCAGGAGCGCACCATCTTCTTCAAGGACGACGGCAACTACAAGACC CGCGCCGAGGTGAAGTTCGAGGGCGACACCCTGGTGAACCGCATCGAGCTGAAG GGCATCGACTTCAAGGAGGACGGCAACATCCTGGGGCACAAGCTGGAGTACAAC TACAACAGCCACAACGTCTATATCATGGCCGACAAGCAGAAGAACGGCATCAAG GTGAACTTCAAGATCCGCCACAACATCGAGGACGGCAGCGTGCAGCTCGCCGAC CACTACCAGCAGAACACCCCCATCGGCGACGGCCCCGTGCTGCTGCCCGACAACC 
ACTACCTGAGCACCCAGTCCGCCCTGAGCAAAGACCCCAACGAGAAGCGCGATC ACATGGTCCTGCTGGAGTTCGTGACCGCCGCCGGGATCACTCTCGGCATGGACGA GCTGTACAAGTCCGGACTCAGATCTCGAGCTCAAGCTTCGAATTCTGCAGTCGAC GGTACCGCGGGCCCGGGATCCACCGGATCTAGATAACTGATCATAATCAGCCATA CCACATTTGTAGAGGTTTTACTTGCTTTAAAAAACCTCCCACACCTCCCCCTGAAC CTGAAACATAAAATGAATGCAATTGTTGTTGTTAACTTGTTTATTGCAGCTTATAA TGGTTACAAATAAAGCAATAGCATCACAAATTTCACAAATAAAGCATTTTTTTCA CTGCATTCTAGTTGTGGTTTGTCCAAACTCATCAATGTATCTTAACGCGTAAATTG TAAGCGTTAATATTTTGTTAAAATTCGCGTTAAATTTTTGTTAAATCAGCTCATTT TTTAACCAATAGGCCG

$*=$ phosphorothioate bond

>CMV-Kozak-FLAG

CTTTCCTGCGTTATCCCCTGATTCTGTGGATAACCGTATTACCGCCATGCATTAGT TATTAATAGTAATCAATTACGGGGTCATTAGTTCATAGCCCATATATGGAGTTCC GCGTTACATAACTTACGGTAAATGGCCCGCCTGGCTGACCGCCCAACGACCCCCG CCCATTGACGTCAATAATGACGTATGTTCCCATAGTAACGCCAATAGGGACTTTC CATTGACGTCAATGGGTGGAGTATTTACGGTAAACTGCCCACTTGGCAGTACATC AAGTGTATCATATGCCAAGTACGCCCCCTATTGACGTCAATGACGGTAAATGGCC CGCCTGGCATTATGCCCAGTACATGACCTTATGGGACTTTCCTACTTGGCAGTAC ATCTACGTATTAGTCATCGCTATTACCATGGTGATGCGGTTTTGGCAGTACATCAA TGGGCGTGGATAGCGGTTTGACTCACGGGGATTTCCAAGTCTCCACCCCATTGAC GTCAATGGGAGTTTGTTTTGGCACCAAAATCAACGGGACTTTCCAAAATGTCGTA ACAACTCCGCCCCATTGACGCAAATGGGCGGTAGGCGTGTACGGTGGGAGGTCT ATATAAGCAGAGCTGGTTTAGTGAACCGTCAGATCCGCTAGCGCTACCGGTCGCC ACCATGGTGGACTACAAAGACGATGACGATAA*A

$*$ = phosphorothioate bond

>NNN-GFP-SV40 PAS

NNNATGGTGAGCAAGGGCGAGGAGCTGTTCACCGGGGTGGTGCCCATCCTGGTC GAGCTGGACGGCGACGTAAACGGCCACAAGTTCAGCGTGTCCGGCGAGGGCGAG GGCGATGCCACCTACGGCAAGCTGACCCTGAAGTTCATCTGCACCACCGGCAAGC TGCCCGTGCCCTGGCCCACCCTCGTGACCACCCTGACCTACGGCGTGCAGTGCTT CAGCCGCTACCCCGACCACATGAAGCAGCACGACTTCTTCAAGTCCGCCATGCCC GAAGGCTACGTCCAGGAGCGCACCATCTTCTTCAAGGACGACGGCAACTACAAG ACCCGCGCCGAGGTGAAGTTCGAGGGCGACACCCTGGTGAACCGCATCGAGCTG AAGGGCATCGACTTCAAGGAGGACGGCAACATCCTGGGGCACAAGCTGGAGTAC AACTACAACAGCCACAACGTCTATATCATGGCCGACAAGCAGAAGAACGGCATC AAGGTGAACTTCAAGATCCGCCACAACATCGAGGACGGCAGCGTGCAGCTCGCC 
GACCACTACCAGCAGAACACCCCCATCGGCGACGGCCCCGTGCTGCTGCCCGAC AACCACTACCTGAGCACCCAGTCCGCCCTGAGCAAAGACCCCAACGAGAAGCGC GATCACATGGTCCTGCTGGAGTTCGTGACCGCCGCCGGGATCACTCTCGGCATGG ACGAGCTGTACAAGTCCGGACTCAGATCTCGAGCTCAAGCTTCGAATTCTGCAGT CGACGGTACCGCGGGCCCGGGATCCACCGGATCTAGATAACTGATCATAATCAG CCATACCACATTTGTAGAGGTTTTACTTGCTTTAAAAAACCTCCCACACCTCCCCC TGAACCTGAAACATAAAATGAATGCAATTGTTGTTGTTAACTTGTTTATTGCAGCT TATAATGGTTACAAATAAAGCAATAGCATCACAAATTTCACAAATAAAGCATTTT TTTCACTGCATTCTAGTTGTGGTTTGTCCAAACTCATCAATGTATCTTAACGCGTA AATTGTAAGCGTTAATATTTTGTTAAAATTCGCGTTAAATTTTTGTTAAATCAGCT CATTTTTTAACCAATAGGCCG

$>$ CMV-GAL4-XTEN-<NNK $\rangle_{15}$

TACGGTTCCTGGCCTTTTGCTGGCCTTTTGCTCACATGGCTCGACAGATCTTCAAT ATTGGCCATTAGCCATATTATTCATTGGTTATATAGCATAAATCAATATTGGCTAT TGGCCATTGCATACGTTGTATCTATATCATAATATGTACATTTATATTGGCTCATG TCCAATATGACCGCCATGTTGGCATTGATTATTGACTAGTTATTAATAGTAATCAA TTACGGGGTCATTAGTTCATAGCCCATATATGGAGTTCCGCGTTACATAACTTAC GGTAAATGGCCCGCCTGGCTGACCGCCCAACGACCCCCGCCCATTGACGTCAATA ATGACGTATGTTCCCATAGTAACGCCAATAGGGACTTTCCATTGACGTCAATGGG TGGAGTATTTACGGTAAACTGCCCACTTGGCAGTACATCAAGTGTATCATATGCC AAGTCCGCCCCCTATTGACGTCAATGACGGTAAATGGCCCGCCTGGCATTATGCC CAGTACATGACCTTACGGGACTTTCCTACTTGGCAGTACATCTACGTATTAGTCAT CGCTATTACCATGGTGATGCGGTTTTGGCAGTACACCAATGGGCGTGGATAGCGG TTTGACTCACGGGGATTTCCAAGTCTCCACCCCATTGACGTCAATGGGAGTTTGTT TTGGCACCAAAATCAACGGGACTTTCCAAAATGTCGTAACAACTGCGATCGCCCG CCCCGTTGACGCAAATGGGCGGTAGGCGTGTACGGTGGGAGGTCTATATAAGCA GAGCTCGTTTAGTGAACCGTCAGATCACTAGAAGCTTTATTGCGGTAGTTTATCA CAGTTAAATTGCTAACGCAGTCAGTGCTTCTGACACAACAGTCTCGAACTTAAGC TGCAGTGACTCTCTTAAGGTAGCCTTGCAGAAGTTGGTCGTGAGGCACTGGGCAG GTAAGTATCAAGGTTACAAGACAGGTTTAAGGAGACCAATAGAAACTGGGCTTG TCGAGACAGAGAAGACTCTTGCGTTTCTGATAGGCACCTATTGGTCTTACTGACA TCCACTTTGCCTTTCTCTCCACAGGTGTCCACTCCCAGTTCAATTACAGCTCTTAA GGCTAGAGTACTTAATACGACTCACTATAGGCTAGCCAGCTTGAAGCAAGCCTCC TGAAAGATGAAGCTACTGTCTTCTATCGAACAAGCATGCGATATTTGCCGACTTA AAAAGCTCAAGTGCTCCAAAGAAAAACCGAAGTGCGCCAAGTGTCTGAAGAACA ACTGGGAGTGTCGCTACTCTCCCAAAACCAAAAGGTCTCCGCTGACTAGGGCACA TCTGACAGAAGTGGAATCAAGGCTAGAAAGACTGGAACAGCTATTTCTACTGATT TTTCCTCGAGAAGACCTTGACATGATTTTGAAAATGGATTCTTTACAGGATATAA AAGCATTGTTAACAGGATTATTTGTACAAGATAATGTGAATAAAGATGCCGTCAC AGATAGATTGGCTTCAGTGGAGACTGATATGCCTCTAACATTGAGACAGCATAGA ATAAGTGCGACATCATCATCGGAAGAGAGTAGTAACAAAGGTCAAAGACAGTTG ACTGTATCGCCGGAATTCCCGGGGATCCGTCGACTTGACGCGTTGATATCATCTA 
GAAGCGGCAGCGAGACTCCCGGGACCTCAGAGTCCGCCACACCCGAATCCNNKN NKNNKNNKNNKNNKNNKNNKNNKNNKNNKNNKNNKNNKNNK

$>$ SV40 PAS

CTCTAGATAACTGAGGTACCTGAATAACTAAGGCCGCTTCCCTTTAGTGAGGGTT

AATGCTTCGAGCAGACATGATAAGATACATTGATGAGTTTGGACAAACCACAACT AGAATGCAGTGAAAAAAATGCTTTATTTGTGAAATTTGTGATGCTATTGCTTTATT TGTAACCATTATAAGCTGCAATAAACAAGTTAACAACAACAATTGCATTCATTTT ATGTTTCAGGTTCAGGGGGAGATGTGGGAGGTTTTTTAAAGCAAGTAAAACCTCT ACAAATGTGGTAAAATCCGATAAGGATCGATCCGGGCTGGCGTAATAGCGAAGA GGCCCGCACCGATCGCCCTTCCCAACAGTTGCGCAGCCTGAATGGCGAATGGACG CGCCCTGTAGCGGCGCATTAAGCGCGGCGGGTGTGGTGGTTACGCGCAGCGTGA CCGCTACACTTGCCAGCGCCCTAGCGCCCGCTCCTTTCGCTTTCTTCCCTTCCTTTC ACGCCACGTTCGCCGGCTTTCCCCGTCAAGCTCTAAATCGGGGGCTCCCTTTAGG GTTCCGATTTAGTGCTTTACGGCACCTCGACCCCAAAAAACTTGATTAGGGTGAT GGTTCACGTAGTGGGCCATCGCCCTGATAGACGGTTTTTCGCCCTTTGACGTTGG AGTCCACGTTCTTTAATAGTGGACTCTTGTTCCAAACTGGAACAACACTCAACCC TATCTCGGTCTATTCTTTTGATTTATAAGGGATTTTGCCGATTTCGGCCTATTGGTT AAAAAATGAGCTGATTTAACAAAAATTTAACGCGAATTTTAACAAAATATTAACG CTTACAATTTCCTGATGCGGTAT 